\title{
Comparison of phase-field models for surface diffusion
}

\author{
Clemens Gugenberger \\ Institut für Festkörperforschung, Forschungszentrum Jülich, D-52425 Jülich, Germany \\ Robert Spatschek \\ Center for Interdisciplinary Research on Complex Systems, Northeastern University, Boston, Massachusetts 02115, USA \\ and Institut für Festkörperforschung, Forschungszentrum Jülich, D-52425 Jülich, Germany \\ Klaus Kassner \\ Institut für Theoretische Physik, Otto-von-Guericke-Universität Magdeburg, Postfach 4120, D-39016 Magdeburg, Germany
}

(Received 12 November 2007; revised manuscript received 10 May 2008; published 21 July 2008)

\begin{abstract}
The description of surface-diffusion controlled dynamics via the phase-field method is less trivial than it appears at first sight. A seemingly straightforward approach from the literature is shown to fail to produce the correct asymptotics, albeit in a subtle manner. Two models are constructed that approximate known sharpinterface equations without adding undesired constraints. Numerical simulations of the standard and a more sophisticated model from the literature as well as of our two models are performed to assess the relative merits of each approach. The results suggest superior performance of the models in at least some situations.
\end{abstract}

DOI: 10.1103/PhysRevE.78.016703

PACS number(s): 02.70.-c, 68.35.Fx, 47.20.Hw

\section{INTRODUCTION}

For a large class of pattern-forming systems, the essential dynamics to be understood and described is that of an interface between two phases. Mathematically speaking, part of the problem to be solved consists of determining the position of the interface as a function of time, i.e., is a free or moving-boundary problem.

During the past decades, phase field modeling has emerged as a powerful tool to tackle this kind of problem. Here, information on the interface position is present implicitly, given either as a level set of a particular value of the phase field (in two-phase models) or by equality of the phase-field values for different phases (in multiphase models), and can be recovered by computation of the appropriate level set at only those times when knowledge of the position is desired. The phase-field description is asymptotic, it does not yield an exact representation of the interface continuum problem, hence an analytic error has to be controlled besides the numerical one in quantitative modeling $[1,2]$.

Most phase-field models deal with nonconservative interface dynamics, for example, in the description of crystal growth, where a particle reservoir is provided by either the melt that is in contact with the solid or the adatom phase on a vicinal surface.

An interesting application, however, is the Asaro-TillerGrinfeld (ATG) instability [3-7], which is an instability with respect to material transport driven by elastic energy; interest initially focused on transport by surface diffusion, which leads to conserved dynamics. This was the case in the first paper by Asaro and Tiller [7], but also in the first numerical simulations by sharp-interface continuum models [8], preceding computations of the instability under transport by melting-crystallization [9].

The situation reversed when the phase-field method was employed for the first time to compute the ATG instability $[3,4]$. Here, all the early works considered a nonconserved phase-field $[3-5,10]$. Only recently has surface diffusion been considered in phase-field models treating elastically stressed materials $[11,12]$. This difference in preferences when modeling either on the basis of a sharp-interface model or using a phase field may be due to the fact that writing down a nonconservative and a conservative model is equally simple in the former case, whereas it is less straightforward to write down the conservative model within the phase-field approach than the nonconservative one.

This is not to say that phase-field models with a conservation law for the phase field have not been considered at all. There is an early paper by Caginalp [13], in which he studies the modifications introduced into a solidification model by making the phase field a conserved order parameter, but this model is not intended to describe surface diffusion. Starting from a Cahn-Hilliard equation with a concentration dependent mobility, Cahn et al. [14] obtained an interface equation with the normal velocity proportional to the Laplacian of the mean curvature. It then appears as if all phase-field models with surface diffusion should be derivable on the basis of similar considerations. Indeed, comparable models have been applied in the simulation of electromigration and voiding in thin metal films $[15,16]$. These two models are slightly different, but the difference is not crucial and all previous models except the one given in [11] seem to suffer from the same problem, to be discussed in the following.

As we shall see, it is quite easy to set up a conservative phase-field model; but it is more difficult to obtain the correct asymptotics describing surface diffusion as given by the desired sharp-interface limit. Past models such as the ones presented in $[12,14-16]$ while asymptotically producing a set of equations containing the desired limit equations, include an additional restriction, i.e., they have one equation too many, a fact that seems to have been overlooked so far. In [11], this restriction is not present, but the authors consider their improvement only a stabilizing element, not changing the asymptotics, whereas what they have achieved in reality is superior asymptotic behavior. Because the flaw of the faulty models is subtle, it is not a priori clear how adversely 
the undesired restriction will affect their behavior. Therefore numerical simulations are necessary to assess their respective virtues and drawbacks.

The purpose of the present paper is to demonstrate the overlooked restriction, to explore an alternative approach to phase-field modeling of surface diffusion, to derive additional models not having the aforementioned flaw, and finally, to compare the different models numerically.

To render things as simple as possible, we will restrict ourselves to two dimensions and give analytic derivations only for purely surface-diffusion-driven motion, i.e., the coupling to a destabilizing process such as elastic relaxation or electromigration will not be considered in the asymptotic analysis. The fully three-dimensional model including elastic energy and thus describing the ATG instability has been given in [17], a paper with lower pedagogical ambitions than this one. In simulations, we will consider both surface diffusion and elastic degrees of freedom, i.e., the ATG instability, to be able to make comparisons for stable and unstable situations.

The paper is organized as follows. In Sec. II, the sharpinterface model to be approximated by the phase-field equations is specified. The nonconservative case will be discussed for reference purposes. Section III then presents the standard approach that previously was supposed to reduce to the correct limit and pinpoints the oversight in existing asymptotic analyses. An alternative approach is presented in Sec. IV, failing for complementary reasons. By appropriate combination of the ideas from both approaches, two phase-field models will be given in Sec. V producing the correct asymptotic behavior. In Sec. VI, comparisons of the different models will be performed via numerical simulation for a number of pertinent situations. Finally, some conclusions to be drawn from both analytic and numerical calculations will be discussed.

\section{SHARP-INTERFACE MODEL FOR MOTION INDUCED BY CURVATURE}

In the simplest case, where surface energy is the only relevant quantity determining the motion of an interface, the local chemical potential difference between the solid and the second phase [liquid, gas (vapor), or vacuum] at the interface may be written

$$
\delta \mu=\frac{1}{\rho_{s}} \gamma \kappa,
$$

where $\rho_{s}$ is the density of the solid phase, $\gamma$ the (isotropic) surface tension, and $\kappa$ the curvature [in three dimensions (3D), the mean curvature]. A positive curvature corresponds to a locally convex solid phase, a negative one to a locally concave solid.

In order to determine the evolution of an unstable state, some dynamical law governing its motion must be stated. If a particle reservoir is present and the interface is rough, the normal velocity $v_{n}$ of the interface will be proportional to the chemical potential difference:

$$
v_{n}=-k_{v} \delta \mu,
$$

where $k_{v}$ is a mobility and the normal points from the solid into the second phase.

On the other hand, for material transport by surface diffusion, the driving force is the gradient of the chemical potential along the surface, producing a surface current $j \propto$ $-\nabla_{s} \delta \mu\left(\nabla_{s}\right.$ is the surface gradient), which leads to a dynamical law of the form

$$
v_{n}=M_{s} \Delta_{s} \delta \mu=M_{s} \frac{\partial^{2} \delta \mu}{\partial s^{2}},
$$

where $\Delta_{s}$ is the Laplace-Beltrami operator on the surface, reducing to a double derivative with respect to the arclength for a one-dimensional interface, and $M_{s}$ a mobility coefficient (dimensionally different from the mobility $k_{v}$ ), assumed constant here.

A linear stability analysis of a planar interface is readily performed, writing

$$
\zeta(x, t)=\zeta_{0}+\epsilon \zeta_{1} e^{\mathrm{i} k x+\omega t},
$$

where $\zeta_{0}$ is the constant position of the unperturbed interface, $x$ the Cartesian coordinate parallel to it, and $\epsilon$ a small parameter used to keep track of orders of the perturbation expansion, and one obtains the dispersion relations

$$
\omega=-\frac{k_{v} \gamma}{\rho_{s}} k^{2} \equiv-K k^{2}
$$

for the nonconservative case and

$$
\omega=-\frac{M_{s} \gamma}{\rho_{s}} k^{4} \equiv-M k^{4}
$$

for the conservative case, respectively. For brevity, we have defined kinetic coefficients $K$ and $M$, which allows us to avoid carrying along the factor $\gamma / \rho_{s}$ all the time.

The two models for motion by curvature considered here are given by Eqs. (1) and (2) on the one hand and Eqs. (1) and (3) on the other, describing the nonconservative and conservative cases, respectively. A phase-field model trying to represent these dynamics should converge to the appropriate set of these sharp-interface equations in the limit of asymptotically small interface width, eventually also with couplings to other fields.

\section{SCALAR-MOBILITY PHASE-FIELD MODEL}

Before considering the structure of previous phase-field models attempting to capture surface diffusion dynamics, let us briefly recall the phase-field model for nonconserved order parameter $\phi$. This can be written [5]

$$
\frac{\partial \phi}{\partial t}=K\left(\nabla^{2} \phi-\frac{2}{W^{2}} f^{\prime}(\phi)\right),
$$

where $f(\phi)=\phi^{2}(1-\phi)^{2}$ is the usual double-well potential describing two-phase equilibrium. $\phi$ varies between 0 , corresponding to the nonsolid phase, and 1, corresponding to the solid; $W$ measures the width of the transition region between 
the two phases, i.e., it may be interpreted as an interface thickness. A prime denotes a derivative with respect to the argument.

The standard approach to a phase-field description of surface diffusion, as proposed in [12,14-16], is then to prepend the right-hand side of Eq. (7) with a differential operator corresponding to the divergence of a gradient multiplied by a phase-field dependent mobility, i.e., Eq. (7) becomes replaced with

$$
\begin{gathered}
\frac{\partial \phi}{\partial t}=-\nabla \cdot \mathbf{j}, \\
\mathbf{j}=-\tilde{M} \nabla \frac{1}{W^{2}} \delta \tilde{\mu}\left(\nabla^{2} \phi, \phi\right), \\
\delta \tilde{\mu}\left(\nabla^{2} \phi, \phi\right) \equiv-W^{2} \nabla^{2} \phi+2 f^{\prime}(\phi),
\end{gathered}
$$

where $\tilde{M}$ is a scalar function of either $\phi[12,14,16]$ or $W \nabla \phi$ [15], chosen such that the mobility tends to zero far from the interface: $\tilde{M}(\phi, W \nabla \phi) \rightarrow 0$ for $\phi \rightarrow 0$ and $\phi \rightarrow 1 . \delta \tilde{\mu}$ is a nondimensionalized chemical potential difference. We will refer to the model described by Eqs. (8) as the scalarmobility model or briefly SM model in the following.

At this point, a few remarks are in order. First, the field $\phi$ is the density of a conserved quantity by construction, since the right-hand side of Eq. (8) is written as a divergence. This is true for any (nonsingular) form of the mobility function $\tilde{M}$. Second, $\delta \tilde{\mu}$ becomes zero for $\phi \rightarrow 0$ and $\phi \rightarrow 1$, meaning that there is no diffusion in the bulk anyway. One might therefore wonder whether it is really necessary to choose a mobility that goes to zero in the bulk. The conservation law plus the absence of diffusion far from the interface should suffice to restrict transport to diffusion along the interface. In fact, we shall see that essentially the same asymptotic results are obtained no matter what the form of $\tilde{M}$, the only conditions to be imposed being positivity (for almost all values of $\phi$ or $\nabla \phi)$ and boundedness. It is just easier to derive them if it is in addition assumed that $\tilde{M}$ vanishes in the bulk. On the other hand, it will turn out that if a restriction imposed by the asymptotics is removed (or not yet satisfied in the temporal evolution of the system), $\tilde{M}$ has to decay sufficiently fast inside the bulk for the limit to make sense. This may be relevant for the behavior of the model before it reaches its asymptotic state.

Finally, the issue at present is not so much whether the dynamics is conservative but whether it does reduce to the sharp-interface model of Sec. II in the limit of an asymptotically vanishing interface thickness. To investigate this, we have to explicitly carry out the asymptotic analysis.

\section{A. Local coordinate system}

The basic idea of the analysis is to expand all dynamical quantities in terms of the small parameter $W$ describing the interface thickness, to solve for the phase field, and to use the solution to eliminate its explicit appearance from the equations. To this end, the domain of definition of the field is divided into an outer region, where gradients of fields can be considered to be of order one and an inner region (close to the interface), where these gradients are of order $1 / W$. The expansion in powers of $W$ is rather straightforward in the outer domain, Eq. (8) can be taken as a starting point directly. As to the inner domain (and its matching with the outer region), it is useful to first transform to coordinates adapted to its geometry. Therefore local coordinates $r$ and $s$ are introduced with $r$ orthogonal to the interface [defined as the level set corresponding to $\phi(x, y, t)=1 / 2]$ and $s$ tangential to it. $r$ is the signed distance from the interface and will be rescaled by a stretching transformation $r=W \rho$ to make explicit the $W$ dependence for the expansion, $s$ is the arclength of the interface curve. Inner and outer solutions must satisfy certain matching conditions due to the requirement that they agree in the combined limit $W \rightarrow 0, \rho \rightarrow \pm \infty$, $r \rightarrow 0$. These conditions are given in Appendix A.

To obtain a set of basis vectors for our local coordinate system, we first write

$$
\mathbf{r}=\mathbf{R}(s)+r \mathbf{n}(s),
$$

where $\mathbf{r}$ is the position vector of a point near the interface, $\mathbf{R}$ the position of the interface itself, and $\mathbf{n}$ the normal vector on it (oriented the same way as in the sharp-interface model, i.e., pointing out of the solid).

Given the coordinates, it is a trivial matter to write down a coordinate basis

$$
\begin{gathered}
\mathcal{E}_{r} \equiv \frac{\partial \mathbf{r}}{\partial r}=\mathbf{n}(s), \\
\mathcal{E}_{s} \equiv \frac{\partial \mathbf{r}}{\partial s}=\frac{\partial \mathbf{R}}{\partial s}+r \frac{\partial \mathbf{n}}{\partial s}=(1+r \kappa) \mathbf{t},
\end{gathered}
$$

which is orthogonal. (This is no longer automatically true in $3 \mathrm{D}[17]$.) $\mathbf{t}=\partial \mathbf{R} / \partial s$ is the unit tangent vector to the interface, and $\partial \mathbf{n} / \partial s=\kappa \mathbf{t}$ is one of the Frenet formulas [18], specialized to two dimensions.

From Eq. (10), we first obtain the metric coefficients $g_{\alpha \beta}=\mathcal{E}_{\alpha} \mathcal{E}_{\beta}$, where $\alpha, \beta \in\{r, s\}$. The metric tensor reads

$$
\left(g_{\alpha \beta}\right)=\mathbf{g}=\left(\begin{array}{cc}
1 & 0 \\
0 & (1+r \kappa)^{2}
\end{array}\right),
$$

its determinant is

$$
g \equiv \operatorname{det} \mathbf{g}=(1+r \kappa)^{2},
$$

hence $\sqrt{g}=1+r \kappa$ (using the locality of $r$ to ascertain $r \kappa<1$ ), and the contravariant components of the metric tensor are obtained as

$$
\left(g^{\alpha \beta}\right)=\mathbf{g}^{-1}=\left(\begin{array}{cc}
1 & 0 \\
0 & (1+r \kappa)^{-2}
\end{array}\right) .
$$

From now on, we use the Einstein summation convention for pairs of covariant and contravariant indices. The vectors of the reciprocal basis are obtained from $\mathcal{E}^{\alpha}=g^{\alpha \beta} \mathcal{E}_{\beta}$ :

$$
\mathcal{E}^{r}=\boldsymbol{\nabla} r=\mathbf{n}(s),
$$




$$
\mathcal{E}^{s}=\nabla s=\frac{1}{\sqrt{g}} \mathbf{t},
$$

The gradient and divergence read

$$
\begin{gathered}
\boldsymbol{\nabla}=\mathcal{E}^{\alpha} \partial_{\alpha}, \\
\boldsymbol{\nabla} \cdot \mathbf{A}=\frac{1}{\sqrt{g}} \partial_{\alpha}\left(\sqrt{g} g^{\alpha \beta} A_{\beta}\right) .
\end{gathered}
$$

Note that on the interface, the covariant component $A_{r}$ is equal to the normal component $A_{n}$, but that $A_{s}$ is related to the tangential component $A_{t}$ by $A_{s}=\sqrt{g} A_{t}$, because $\mathcal{E}^{s}$ is not normalized to one.

In the following, we will denote inner quantities by the uppercase letter corresponding to the lowercase letter denoting the outer quantity, whenever this is meaningful. Since the interface will move in general and the coordinates $r$ and $s$ are defined with respect to the interface, there is also a transformation rule for the time derivative:

$$
\partial_{t} f(x, z, t)=\partial_{t} F(r, s, t)-\mathbf{v} \cdot \nabla F(r, s, t),
$$

where $\mathbf{v}(s, t)$ is the interface velocity. Equation (17) exhibits that the time derivative in the comoving frame is a material derivative.

\section{B. Inner equations}

To render the scales of the different terms more visible, we rewrite Eqs. (15) and (16) in the form

$$
\begin{gathered}
\boldsymbol{\nabla}=\frac{1}{W} \mathbf{n} \partial_{\rho}+\frac{1}{\sqrt{g}} \mathbf{t} \partial_{s}, \\
\boldsymbol{\nabla} \cdot \mathbf{A}=\frac{1}{\sqrt{g}}\left(\frac{1}{W} \partial_{\rho} \sqrt{g} A_{r}+\partial_{s} \frac{1}{\sqrt{g}} A_{s}\right), \\
\sqrt{g}=1+W \rho \kappa .
\end{gathered}
$$

Assuming, without loss of generality, that the tangential velocity of the interface vanishes, Eq. (8) takes the following form:

$$
\begin{gathered}
\partial_{t} \Phi-\frac{1}{W} v_{n} \partial_{\rho} \Phi=\nabla \cdot \tilde{M} \nabla \frac{1}{W^{2}} \delta \tilde{\mu}\left(\nabla^{2} \Phi, \Phi\right), \\
\delta \tilde{\mu}\left(\nabla^{2} \Phi, \Phi\right)=-\frac{1}{\sqrt{g}} \partial_{\rho} \sqrt{g} \partial_{\rho} \Phi-W^{2} \frac{1}{\sqrt{g}} \partial_{s} \frac{1}{\sqrt{g}} \partial_{s} \Phi+2 f^{\prime}(\Phi),
\end{gathered}
$$

with

$$
\boldsymbol{\nabla} \cdot \tilde{M} \boldsymbol{\nabla}=\frac{1}{W^{2}} \frac{1}{\sqrt{g}} \partial_{\rho} \sqrt{g} \tilde{M} \partial_{\rho}+\frac{1}{\sqrt{g}} \partial_{s} \frac{1}{\sqrt{g}} \tilde{M} \partial_{s} .
$$

Hence the leading term of the inner Eq. (21) with the differential operator given by Eq. (22) is of order $W^{-4}$.

\section{Expansions, matched asymptotic analysis}

To solve the outer and inner equations successively, we expand the phase field in both the outer and inner domains in powers of $W$,

$$
\phi(x, z, t)=\phi^{(0)}(x, z, t)+W \phi^{(1)}(x, z, t)+W^{2} \phi^{(2)}(x, z, t) \cdots
$$

and

$$
\Phi(r, s, t)=\Phi^{(0)}(r, s, t)+W \Phi^{(1)}(r, s, t)+W^{2} \Phi^{(2)}(r, s, t) \cdots .
$$

We now proceed solving the outer and inner equations order by order.

\section{Leading order}

The leading-order outer equation for $\phi$, which follows immediately from Eq. (8), is

$$
\boldsymbol{\nabla} \cdot \tilde{M} \boldsymbol{\nabla} f^{\prime}\left(\phi^{(0)}\right)=0,
$$

which is to be supplemented with the boundary conditions $\phi^{(0)}=1$ and $\phi^{(0)}=0$ at infinity in the regions where the system is solid and nonsolid, respectively. If we regard Eq. (25) as a partial differential equation for the function $f^{\prime}\left(\phi^{(0)}\right)$ (rather than for $\phi^{(0)}$ itself), this boundary condition translates into $f^{\prime}\left(\phi^{(0)}\right) \rightarrow 0$ as $|\mathbf{r}| \rightarrow \infty$. The new boundary condition is valid everywhere at infinity (except possibly in a region with size of order $W)$. For general $\tilde{M}(\phi, W \nabla \phi)$, the partial differential equation (25) is of course nonlinear. Nevertheless, it can be shown to have the unique solution $f^{\prime}\left(\phi^{(0)}\right)=0$ under certain mild conditions, if $\tilde{M}$ is positive everywhere, except possibly on a set of measure zero (see Appendix B for a more detailed discussion). From a physical point of view, this is of course the desired solution, as in the sharp interface limit the system must be in one of the stable states $\phi^{(0)}=0$ or $\phi^{(0)}=1$.

It is then seen by inspection that the outer equation is indeed solved to all orders by the solution under discussion. With the usual construction of coupling terms to, say, mechanical or electrical degrees of freedom $[4,15]$, this remains true for the full phase-field model including the coupling, as these terms are typically made to vanish in the bulk. So our statement, which has some importance as we shall see, is valid beyond the oversimplified "free" model considered here for the purpose of demonstration.

Therefore we have $\phi^{(1)} \equiv 0, \phi^{(2)} \equiv 0$, which provides us with partial boundary conditions for the inner solutions $\Phi^{(1)}$, $\Phi^{(2)}$, and so on (see Appendix A). Moreover, only the inner problem needs to be considered beyond the leading order.

Because $g=1+O(W)$, the leading-order inner problem becomes [see Eqs. (21) and (22)]

$$
\partial_{\rho} \tilde{M}\left(\Phi^{(0)}\right) \partial_{\rho}\left[\partial_{\rho \rho} \Phi^{(0)}-2 f^{\prime}\left(\Phi^{(0)}\right)\right]=0,
$$

where $\partial_{\rho \rho}=\partial_{\rho}^{2}$. At this order, we obtain the expected solution $\Phi^{(0)}=\frac{1}{2}(1-\tanh \rho)$. However, since the proper use of the boundary conditions will turn out to be essential for the higher order equations and in order to show that there are no additional solutions to this fourth-order equation, we give the 
detailed line of argument, to be used also below. The above equation can be integrated once to yield

$$
\partial_{\rho}\left[\partial_{\rho \rho} \Phi^{(0)}-2 f^{\prime}\left(\Phi^{(0)}\right)\right]=\frac{c_{1}(s)}{\tilde{M}\left(\Phi^{(0)}\right)},
$$

where $c_{1}(s)$ is a function of integration. We argue differently, depending on whether $\tilde{M}$ approaches zero for $\rho \rightarrow \pm \infty$, which is the case for the mobilities assumed in $[12,14,16]$, or whether it is just a bounded (and possibly constant) function of $\phi$. In the first case, we may immediately conclude $c_{1}=0$ because the right-hand side of Eq. (27) must not diverge. In the second case, we obtain the same result by integrating Eq. (27) first and invoking the boundary conditions:

$$
\partial_{\rho \rho} \Phi^{(0)}-2 f^{\prime}\left(\Phi^{(0)}\right)=c_{1}(s) \int_{0}^{\rho} \frac{1}{\tilde{M}} d \rho+c_{2}(s) .
$$

Since $\tilde{M}$ is bounded from above and positive, the integral will be larger in magnitude than $\int_{0}^{\rho} 1 /\left(\sup _{\rho} \tilde{M}\right) d \rho$ $=\rho / \sup _{\rho} \tilde{M}$, so the two factors multiplying $c_{1}$ and $c_{2}$ are linearly independent. The left-hand side approaches zero for $\rho \rightarrow \pm \infty$ [the argument will be made more rigorous below in the discussion of $\left.\Phi^{(1)}\right]$, so both $c_{1}$ and $c_{2}$ must be equal to zero. To argue that $c_{2}$ is zero in the case where $\tilde{M} \rightarrow 0$ for $\rho \rightarrow \pm \infty$, we can proceed the same way, except that we have already gotten rid of the term containing $c_{1}$, so the right-hand side of Eq. (28) is $c_{2}$ only.

To summarize, the leading-order inner equation results in

$$
\partial_{\rho \rho} \Phi^{(0)}-2 f^{\prime}\left(\Phi^{(0)}\right)=0
$$

and this provides us with the well-known solution

$$
\Phi^{(0)}=\frac{1}{2}(1-\tanh \rho) .
$$

\section{Next-to-leading order}

The next-to-leading order in Eq. (21) is the order $W^{-3}$. Since the differential operator in front of the chemical potential is of order $W^{-2}$ and the chemical potential multiplied by another factor $W^{-2}$, we must expand $\delta \tilde{\mu}$ up to order $W$. Equation (29) already tells us that $\delta \widetilde{\mu}^{(0)}=0$, so we obtain

$$
\partial_{\rho} \tilde{M}\left(\Phi^{(0)}\right) \partial_{\rho} \delta \tilde{\mu}^{(1)}=0,
$$

from which we get

$$
\partial_{\rho} \delta \tilde{\mu}^{(1)}=\frac{d_{1}(s)}{\tilde{M}\left(\Phi^{(0)}\right)} .
$$

As before, we can immediately conclude from this that $d_{1}$ $=0$, if we assume $\tilde{M}\left(\Phi^{(0)}\right) \rightarrow 0$ for $\Phi^{(0)} \rightarrow 0,1$. For arbitrary but bounded $\tilde{M}$, we invoke the matching conditions (see Appendix A)

$$
\lim _{\rho \rightarrow \pm \infty} \partial_{\rho} \delta \tilde{\mu}^{(1)}=\left.\partial_{r} \delta \tilde{\mu}_{\text {out }}^{(0)}\right|_{ \pm 0}=0
$$

to obtain the same result (where for once we have denoted an outer quantity by a subscript "out").
Integrating once more with respect to $\rho$ and writing out $\delta \tilde{\mu}^{(1)}$, we have

$$
\delta \tilde{\mu}^{(1)}=-\partial_{\rho \rho} \Phi^{(1)}-\kappa \partial_{\rho} \Phi^{(0)}+2 f^{\prime \prime}\left(\Phi^{(0)}\right) \Phi^{(1)}=d_{2}(s) .
$$

Up to this point, there is agreement between this and preceding asymptotic analyses [15], if not in all details of the procedure, so at least in the results.

Let us now try to determine the function of integration $d_{2}(s)$. A priori, there is no reason to use a procedure different from what we have done before. We know the limiting behavior for $\rho \rightarrow \pm \infty$ for two of the four terms on the righthand side of Eq. (34): $\lim _{\rho \rightarrow \pm \infty} \partial_{\rho} \Phi^{(0)}=0$ (which follows from the matching conditions) and $\lim _{\rho \rightarrow \pm \infty} d_{2}(s)=d_{2}(s)$ (because $d_{2}$ is independent of $\rho$ ). Moreover, from the matching conditions, we obtain the limit for $\Phi^{(1)}$,

$$
\Phi^{(1)} \sim \rho \phi^{\prime(0)}( \pm 0)+\phi^{(1)}( \pm 0)=\phi^{(1)}( \pm 0)=0 \quad(\rho \rightarrow \pm \infty) .
$$

The second equality follows from the fact that $\phi^{(0)}=0$ or $\phi^{(0)}=1$, hence its derivative with respect to $r$ vanishes on both sides of the interface; the third equality is a consequence of the fact that $\phi^{(0)}$ solves the outer equation to all orders and hence $\phi^{(1)} \equiv 0$. In addition, it can be shown [17] that if the interaction with mechanical degrees of freedom is included, this will only lead to terms that also vanish in the limit $\rho \rightarrow \pm \infty$.

With three of the four terms in Eq. (34) having a definite limit, we may conclude that the fourth must have a limit as well and obtain

$$
\lim _{\rho \rightarrow \pm \infty}-\partial_{\rho \rho} \Phi^{(1)}=d_{2}(s)
$$

but if this limit exists, it cannot be different from zero: transforming to $\xi=1 / \rho$, we see that $\partial_{\rho \rho} \Phi^{(1)}=\left(\xi^{2} \partial_{\xi}\right)^{2} \Phi^{(1)}$, which implies the asymptotic behavior $\Phi^{(1)} \sim-d_{2} / 2 \xi^{2}(\xi \rightarrow 0)$ and hence the divergence of $\Phi^{(1)}$ as $-d_{2} \rho^{2} / 2$, if $d_{2} \neq 0$. [The same kind of argument can be used to show that the left-hand side of Eq. (28) goes to zero, even though the matching conditions do not provide a direct expression for $\lim _{\rho \rightarrow \pm \infty} \partial_{\rho \rho} \Phi^{(0)}$.]

The upshot of these detailed considerations is that

$$
d_{2}(s)=\delta \tilde{\mu}^{(1)}=0 .
$$

Previous treatments of the problem did not enter into these considerations. Instead, they only employed the additional condition which follows from Fredholm's alternative, involving the properties of the linear operator

$$
\mathcal{L}=\partial_{\rho \rho}-2 f^{\prime \prime}\left(\Phi^{(0)}\right)
$$

or, equivalently, from multiplication with $\partial_{\rho} \Phi^{(0)}$ and subsequent integration by parts. Doing the same thing, we obtain from Eq. (34)

$$
-\int_{-\infty}^{\infty} \kappa\left(\partial_{\rho} \Phi^{(0)}\right)^{2} d \rho=\int_{-\infty}^{\infty} \partial_{\rho} \Phi^{(0)} d_{2}(s) d \rho=-d_{2}(s),
$$

from which we get, using the tanh solution for $\Phi^{(0)}$, 


$$
d_{2}(s)=\frac{1}{3} \kappa .
$$

Both Eqs. (37) and (40) were derived by valid methods, therefore they should both hold true. Nevertheless, as we shall see shortly, Eq. (37) is a quite undesirable result. This may be the deeper reason why it was so far overlooked and only the analog of Eq. (40) derived. When Eq. (37) is inserted in Eq. (40), it leads to zero curvature at lowest order. In a phase-field model for the ATG instability [17], the same kind of reasoning imposes a relationship between the elastic state of the material and the curvature. In models, where the interaction term is quadratic in $W$ [15], it again imposes the restriction $\kappa=O(W)$. To summarize, in all cases we obtain a restriction on the curvature at lowest order, which means that the phase-field model will not be asymptotic as long as the deviation from this imposed value is not small.

\section{Higher orders}

To see that the model would indeed work if we did not have the restriction (37), let us consider the equations at the next two orders, ignoring for the time being the result $d_{2}$ $=0$. Since both $\delta \widetilde{\mu}^{(0)}(=0)$ and $\delta \tilde{\mu}^{(1)}$ are independent of $\rho$, the first term of the operator (22) does not produce any contribution from these terms in Eq. (21), and the order $W^{-2}$ equation reads

$$
\partial_{\rho} \tilde{M} \partial_{\rho} \delta \tilde{\mu}^{(2)}+\partial_{s} \tilde{M} \partial_{s} \delta \tilde{\mu}^{(0)}=0,
$$

where we can immediately drop the second term because of $\delta \widetilde{\mu}^{(0)}=0$. After two integrations this becomes

$$
\delta \tilde{\mu}^{(2)}=e_{1}(s) \int_{0}^{\rho} \frac{1}{\tilde{M}} d \rho+e_{2}(s) .
$$

If $\tilde{M} \rightarrow 0$ for $\rho \rightarrow \pm \infty$, we immediately find $e_{1}(s)=0$. In the general case, we use the matching conditions [see Eq. (A6)]

$$
\left.\delta \tilde{\mu}^{(2)} \sim \frac{1}{2} \rho^{2} \partial_{r r} \delta \tilde{\mu}_{\mathrm{out}}^{(0)}\right|_{r= \pm 0}+\left.\rho \partial_{r} \delta \tilde{\mu}_{\mathrm{out}}^{(1)}\right|_{r= \pm 0}+\left.\delta \tilde{\mu}_{\mathrm{out}}^{(2)}\right|_{r= \pm 0} .
$$

From Eq. (8), we gather that an expansion of $\delta \widetilde{\mu}_{\text {out }}$ in powers of $W$ will contain three types of terms, the first of which have the form $\nabla^{2} \phi^{(k)}(k=0,1, \ldots)$, while the second contain factors $\phi^{(k)}(k=1,2, \ldots)$, coming from an expansion of $f^{\prime}(\phi)$ about $\phi^{(0)}$, and the third include $f^{\prime}\left(\phi^{(0)}\right)$ alone. All of these terms vanish because $\phi^{(k)}=0$ for $k>0$ and because $f^{\prime}\left(\phi^{(0)}\right)$ $=0$. This is simply a consequence of the fact that the outer equation is solved exactly by $\phi^{(0)}=0$ and $\phi^{(0)}=1$. The "chemical potential" appearing in the phase-field equations needs to be related to the true, i.e., sharp-interface chemical potential only at the interface. In the outer domain, it is zero. We can then conclude from Eq. (43) that $e_{1}(s)=0$ [of course $e_{2}(s)=0$, too, but we shall not make use of that result]

Given that $\delta \tilde{\mu}^{(2)}$ is independent of $\rho$, the inner equation at order $W^{-1}$ takes the form

$$
-v_{n} \partial_{\rho} \Phi^{(0)}=\partial_{\rho} \tilde{M} \partial_{\rho} \delta \tilde{\mu}^{(3)}+\partial_{s} \tilde{M} \partial_{s} \delta \tilde{\mu}^{(1)} .
$$

After integration over $\rho\left(v_{n}\right.$ does not depend on $\left.\rho\right)$ we find

$$
v_{n}=\partial_{s s} \delta \tilde{\mu}^{(1)} \int_{-\infty}^{\infty} \tilde{M}\left(\Phi^{(0)}\right) d \rho+\left.\tilde{M}\left(\Phi^{(0)}\right) \partial_{\rho} \delta \tilde{\mu}^{(3)}\right|_{-\infty} ^{\infty}
$$

Here we can drop the second term on the right-hand side if $\lim _{\rho \rightarrow \pm \infty} \tilde{M}\left(\Phi^{(0)}\right)=0$. Formally setting $\int_{-\infty}^{\infty} \tilde{M}\left(\Phi^{(0)}\right) d \rho=3 M$ and using Eq. (40), we arrive at

$$
v_{n}=M \partial_{s s} \kappa .
$$

Hence Eq. (46) reproduces the sharp-interface limit (3), with the relationship between $M_{s}$ and $M$ defined in Eq. (6).

Finally, $M$ would be infinite for positive functions $\tilde{M}\left(\Phi^{(0)}\right)$ that do not reduce to zero for $\rho \rightarrow \pm \infty$; therefore in the end we would indeed have to require $\tilde{M}\left(\Phi^{(0)}\right)$ to decay far from the interface, if $\delta \tilde{\mu}^{(1)}$ were different from zero. In reality, we do not just have Eq. (46), the equation we want, but in addition Eq. (37), requiring $\delta \widetilde{\mu}^{(1)}=0$ and, consequently

$$
v_{n}=0 .
$$

At first sight, Eqs. (46) and (47) may look like they are contradicting each other, as we can prepare an initial state with arbitrary curvature of the interface, and hence the velocity should be different from zero according to Eq. (46) but equal to zero according to Eq. (47). However, in preparing an arbitrary initial state, we have no certainty that the system will already follow its (lowest-order) asymptotic dynamics. A similar phenomenon happens in all phase-field models when a simulation is started with an initial interface perturbed by white noise. Since the asymptotics of the phasefield equations require curvatures to be smaller than $1 / W$, the initial stage of the dynamics where larger curvatures are present will not be governed by these asymptotics; but in that case the asymptotic behavior is sufficiently robust to keep the initial stage short.

It is instructive to note why the nonconservative model obtained when Eq. (8) is replaced with Eq. (7) does not suffer from a similar difficulty. In that model, the velocity is already determined at the next-to-leading order. Instead of Eq. (34), we get

$$
-v_{n} \partial_{\rho} \Phi^{(0)}=K\left\{\partial_{\rho \rho} \Phi^{(1)}+\kappa \partial_{\rho} \Phi^{(0)}-2 f^{\prime \prime}\left(\Phi^{(0)}\right) \Phi^{(1)}\right\} .
$$

Again we may conclude that all the terms on the right-hand side go to zero as $\rho$ is sent to $\pm \infty$. However, this does not lead to any constraints, since the left-hand side is $\rho$ dependent now and goes to zero as well, satisfying the limit automatically, whereas in the surface-diffusion case, it was a function of $s$ only $\left(d_{2}\right)$ that could be concluded to be equal to zero. So consideration of the limit does not produce anything new here, and the only procedure available to extract information on $v_{n}$ is to use Fredholm's alternative which gives the correct sharp-interface limit.

In the case of the nonconservative model, the introduced chemical potential functional is zero in the bulk just as in the conservative case, but there are no restrictions on its variation near the interface, where it acquires a form tending to a $\delta$ function in the sharp-interface limit. In the conservative model, this is excluded by restrictions on the derivative of the chemical potential with respect to $\rho$, meaning that the latter must be smooth across the interface. Since it is zero off 
the interface, it is zero on it as well. Due to this reason, the phase-field model strictly speaking applies only to the equilibrium limit. Far-from-equilibrium dynamics is not likely to be captured faithfully.

Out of the phase-field models for surface diffusion considered in the literature, the only one that is (apart from our own work [17]) not subject to the criticism offered here seems to be the one given by Rätz, Ribalta, and Voigt [11]. Let us briefly discuss the asymptotics of this model that we will henceforth denote as the $R R V$ model. In their simplest form, i.e., for isotropic surface tension and vanishing kinetic coefficient, the model equations read

$$
\begin{gathered}
\frac{\partial \phi}{\partial t}=-\nabla \cdot \mathbf{j}, \\
\mathbf{j}=-M B(\phi) \nabla \frac{1}{W^{2}} \delta \hat{\mu}\left(\nabla^{2} \phi, \phi\right), \\
\delta \hat{\mu}=\frac{1}{g(\phi)}\left[-W^{2} \nabla^{2} \phi+2 f^{\prime}(\phi)\right],
\end{gathered}
$$

with mobility function $B(\phi)=12 \phi^{2}(1-\phi)^{2}$, double-well potential $f(\phi)=\phi^{2}(1-\phi)^{2}$, and the so-called stabilizing function $g(\phi)=10 \phi^{2}(1-\phi)^{2}$. Here, we have rescaled the equations from [11] so as to obtain the same zeroth-order interface profile as in the SM model (with the original equations, the interface would have one-third of the width of our profile). The clue is here that the additional singular term $1 / g(\phi)$ in the chemical potential combines in a proper way with the decay of the mobility function, hence a constraint in the spirit of Eq. (37) does not exist. The leading-order inner problem becomes Eq. (29) again. At next-to-leading order, we obtain $\delta \widetilde{\mu}^{(1)}=d_{2}(s)$ (as before), but now the chemical potential function is defined differently-it has a prefactor that diverges in the bulk

$$
\delta \widetilde{\mu}^{(1)}=\frac{1}{g\left(\Phi^{(0)}\right)}\left[-\partial_{\rho \rho} \Phi^{(1)}-\kappa \partial_{\rho} \Phi^{(0)}+2 f^{\prime \prime}\left(\Phi^{(0)}\right) \Phi^{(1)}\right] .
$$

[The first-order term due to variation of the denominator vanishes, as it contains the differential expression from the left-hand side of Eq. (29) as a factor.] The numerator of the right-hand side of Eq. (50) goes to zero as $\rho \rightarrow \pm \infty$ but so does the denominator $g\left(\Phi^{(0)}\right)$, which renders $\delta \tilde{\mu}^{(1)}$ indefinite, thus introducing the degree of freedom necessary for a nonzero value $d_{2}(s)$. Multiplying the equation by $g\left(\Phi^{(0)}\right) \partial_{\rho} \Phi^{(0)}$ and integrating with respect to $\rho$ from $-\infty$ to $\infty$, we arrive at

$$
d_{2} \int_{-\infty}^{\infty} g\left(\Phi^{(0)}\right) \partial_{\rho} \Phi^{(0)} d \rho=-\kappa \int_{-\infty}^{\infty}\left(\partial_{\rho} \Phi^{(0)}\right)^{2} d \rho,
$$

where use has been made of the fact that $\partial_{\rho} \Phi^{(0)}$ is a left null eigenvector of the linear operator [inside the brackets in Eq. (50)] acting on $\Phi^{(1)}$. The integrals in Eq. (51) are equal to $-1 / 3$ and $1 / 3$, respectively. Hence $d_{2}=\kappa$.

The steps for the following two orders of $W$ follow precisely the scheme leading from Eq. (41) to Eq. (46), which then yields $v_{n}=M^{*} \partial_{s s} \kappa$, where $M^{*}=\int_{-\infty}^{\infty} M B\left(\Phi^{(0)}\right) d \rho=M$, hence we obtain the desired sharp-interface limit (3).

Note that with this model, it is essential that the mobility function goes to zero off the interface. For the chemical potential $\delta \hat{\mu}$ varies in the bulk [it behaves as $d_{2}(s)$ near the interface], hence diffusion there must be suppressed by a vanishing mobility.

\section{TENSORIAL MOBILITY}

The preceding results raise the question how one could obtain an improved phase-field model giving the correct asymptotic behavior without the unphysical device of rendering the chemical potential indefinite in the bulk.

That the phase-field model given by Eq. (8) does not quite yield the correct asymptotics may be traced back to the fact that the differential operator $\boldsymbol{\nabla} \cdot \tilde{M} \boldsymbol{\nabla}$, prepended to the chemical potential, does not reduce to the surface Laplacian $\Delta_{s}$ in the asymptotic limit. In fact, the second term on the righthand side of Eq. (22) is, up to a factor, the Laplace-Beltrami operator on the surface (for $\rho=0$ ), but the first term, containing derivatives with respect to $\rho$ is orders of magnitude larger, being preceded by a factor of $1 / W^{2}$. As a consequence, the asymptotics must be secured by the full solution of the equation rather than by both the operator and the chemical potential converging to the desired sharp-interface limits.

Realizing this property of the model, it seems natural to modify the differential operator via introduction of an essentially tensorial mobility. As a result, the diffusion tensor will become anisotropic, with zero eigenvalue in one of the three spatial directions, which also seems a physically satisfactory solution to the problem of representing diffusion on a surface. Let us denote by

$$
\hat{\mathbf{n}}=-\frac{\nabla \phi}{|\nabla \phi|}
$$

the normal on the surface $\phi=$ const (for $\phi=1 / 2$, we have $\hat{\mathbf{n}}$ $=\mathbf{n})$, then we expect the operator $\nabla \cdot P \nabla$ with

$$
P=1-\hat{\mathbf{n}}: \hat{\mathbf{n}}
$$

(Cartesian components: $P_{i j}=\delta_{i j}-\hat{n}_{i} \hat{n}_{j}$ ) to reduce to the surface Laplacian asymptotically. A colon is used to designate a dyadic product, so $P$ is a projection operator projecting onto the tangential plane of a level set of $\phi$. The same kind of projection operator has been used to obtain the surface Laplacian from the 3D one in level set approaches, both with static [20] and dynamically evolving surfaces [21].

This then suggests to replace the phase-field Eq. (8) with

$$
\frac{\partial \phi}{\partial t}=M \boldsymbol{\nabla} \cdot(1-\hat{\mathbf{n}}: \hat{\mathbf{n}}) \boldsymbol{\nabla} \frac{1}{W^{2}} \delta \tilde{\mu}\left(\nabla^{2} \phi, \phi\right),
$$

where $\delta \tilde{\mu}$ is unchanged from Eq. (8) but $M$ is a constant mobility now.

However, model (54) fails much more miserably than model (8). The reason is that the zeroth-order solution is not unique. Intuitively, this behavior can be easily understood for a planar interface. Then the equation of motion (54) strictly contains only derivatives of the phase field parallel to the 
interface, and the profile in the perpendicular direction therefore remains completely undetermined.

It can be said that this model fails for reasons complementary to those of the scalar model. Whereas we had one equation too many in that case, adding a constraint to the desired sharp-interface dynamics, now we have one equation too few, as there is nothing in the model fixing $\phi^{(0)}$. If we had the right $\phi^{(0)}$, the tensorial model would work perfectly.

\section{MODIFIED TENSORIAL MOBILITY MODELS WITH CORRECT ASYMPTOTICS}

In order to obtain a model not plagued by either of the disadvantages of the two cases discussed, it appears that it is useful to combine ideas from both. While it is certainly desirable to have a differential operator that itself approaches the surface Laplacian, it should do so only for phase-field functions that have the correct leading-order profile.

\section{A. Locally conservative model}

One way to achieve this goal is to modify $1-\hat{\mathbf{n}}: \hat{\mathbf{n}}$ into

$$
Q \equiv 1-W^{2} \frac{\boldsymbol{\nabla} \phi: \nabla \phi}{4 f(\phi)}=1-\frac{W^{2}(\boldsymbol{\nabla} \phi)^{2}}{4 f(\phi)} \hat{\mathbf{n}}: \hat{\mathbf{n}} .
$$

If we replace the projection operator in Eq. (54) by $Q$, then the outer equation at leading order will have the same differential operator as the scalar model with constant $M$, whereas in the inner region we have $Q=1-\hat{\mathbf{n}}: \hat{\mathbf{n}}+O(W) \hat{\mathbf{n}}: \hat{\mathbf{n}}$ [because Eq. (29) implies $\left.W^{2}(\boldsymbol{\nabla} \phi)^{2}=4 f(\phi)+O(W)\right]$.

A better inner approximation to $1-\hat{\mathbf{n}}: \hat{\mathbf{n}}$ than just $Q$ is provided by a minor modification. Taking $Q$ to some integer power $m$ we get, since $1-\hat{\mathbf{n}}: \hat{\mathbf{n}}$ and $\hat{\mathbf{n}}: \hat{\mathbf{n}}$ are orthogonal projectors:

$$
Q^{m}=1-\hat{\mathbf{n}}: \hat{\mathbf{n}}+O\left(W^{m}\right) \hat{\mathbf{n}}: \hat{\mathbf{n}} .
$$

These considerations lead us to make the ansatz

$$
\begin{gathered}
\frac{\partial \phi}{\partial t}=-M \boldsymbol{\nabla} \cdot \mathbf{j}, \\
\mathbf{j}=-Q^{m} \boldsymbol{\nabla} \frac{1}{W^{2}} \delta \tilde{\mu}\left(\nabla^{2} \phi, \phi\right), \\
\delta \widetilde{\mu} \equiv-W^{2} \nabla^{2} \phi+2 f^{\prime}(\phi)
\end{gathered}
$$

and leave the precise choice of the value of $m$ for later-it will be suggested by the asymptotic analysis.

The corresponding inner equations are

$$
\begin{gathered}
\partial_{t} \Phi-\frac{1}{W} v_{n} \partial_{\rho} \Phi=M \nabla \cdot Q^{m} \nabla \frac{1}{W^{2}} \delta \widetilde{\mu}\left(\nabla^{2} \Phi, \Phi\right), \\
\delta \widetilde{\mu}\left(\nabla^{2} \Phi, \Phi\right)=-\frac{1}{\sqrt{g}} \partial_{\rho} \sqrt{g} \Phi_{, \rho}-W^{2} \frac{1}{\sqrt{g}} \partial_{s} \frac{1}{\sqrt{g}} \Phi_{, s}+2 f^{\prime}(\Phi),
\end{gathered}
$$

where $\Phi_{s}$ is shorthand for $\partial_{s} \Phi$ and

$$
\begin{aligned}
\boldsymbol{\nabla} \cdot Q^{m} \boldsymbol{\nabla}= & \frac{1}{\sqrt{g}} \partial_{\nu} \sqrt{g} g^{\nu \mu}\left\{\partial_{\mu}-\left[1-\left(1-\frac{W^{2}(\boldsymbol{\nabla} \Phi)^{2}}{4 f(\Phi)}\right)\right]\right. \\
& \left.\times \frac{1}{(\boldsymbol{\nabla} \Phi)^{2}} \Phi_{, \mu} g^{\alpha \beta} \Phi_{, \alpha} \partial_{\beta}\right\} .
\end{aligned}
$$

\section{Leading order}

In the outer equations, $Q$ becomes the identity operator to leading order, i.e., $Q^{(0)}\left(\phi^{(0)}\right)=1$, and at the lowest order in $W$, we have

$$
\nabla^{2} f^{\prime}\left(\phi^{(0)}\right)=0,
$$

a Laplace equation that we know to be uniquely solvable for $f^{\prime}\left(\phi^{(0)}\right)$ with Dirichlet boundary conditions at infinity. This boundary condition is even homogeneous (except possibly in a part of the boundary region at infinity having a size of order $W$ ), leading to the unique solution $f^{\prime}\left(\phi^{(0)}\right) \equiv 0$. This leaves us with the three possibilities $\phi^{(0)}=0, \frac{1}{2}, 1$, of which $\phi^{(0)}=0$ or $\phi^{(0)}=1$ are realized, according to the particular boundary condition on $\phi^{(0)}$.

Again, $\phi=0$ and 1 are solutions to the outer problem at all orders of $W$. Admittedly, the operator $Q$ becomes indefinite at order $W^{2}$ for $\phi=0$ and 1 [because of the denominator $f(\phi)]$, but this does not matter, since the expression for $\delta \tilde{\mu}$ alone is zero already at $\phi=0$ and 1 .

The leading-order inner equation reads $[g=1+O(W)]$

$$
\partial_{\rho}\left[1-\frac{\left(\Phi_{, \rho}^{(0)}\right)^{2}}{4 f\left(\Phi^{(0)}\right)}\right]^{m} \partial_{\rho}\left[\partial_{\rho \rho} \Phi^{(0)}-2 f^{\prime}\left(\Phi^{(0)}\right)\right]=0 .
$$

Clearly, this is solved by $\Phi^{(0)}=\frac{1}{2}(1-\tanh \rho)$, which makes both the expression in brackets and in large parentheses vanish. If we require $m$ to be even, this solution is moreover unique (up to translations, which are eliminated by the requirement that the interface be at $\rho=0$ ). For as soon as we assume $\left(\Phi_{\rho \rho}^{(0)}\right)^{2} \neq 4 f\left(\Phi^{(0)}\right)$, the $m$ th power of the bracket expression will be positive, allowing us to use similar arguments as in Sec. III C between Eqs. (26) and (29) to prove that $\partial_{\rho \rho} \Phi^{(0)}-2 f^{\prime}\left(\Phi^{(0)}\right)=0$, and hence the bracket expression must be zero, contrary to our assumption. Thus we do get a definite solution for $\Phi^{(0)}$ from the inner equation, which moreover shows that at leading order of the inner expansion the second-order $\rho$ derivatives of the operator $\boldsymbol{\nabla} \cdot Q^{m} \boldsymbol{\nabla}$ cancel each other.

\section{Next-to-leading order}

To simplify computations at the next order, we first expand $\boldsymbol{\nabla} \cdot Q^{m} \boldsymbol{\nabla}$ up to formal order $W^{0}$. This produces

$$
\begin{aligned}
\boldsymbol{\nabla} \cdot Q^{m} \boldsymbol{\nabla}= & \frac{1}{\sqrt{g}} \partial_{s} \frac{1}{\sqrt{g}} \partial_{s}+\frac{1}{\sqrt{g}} \frac{1}{W^{2}} \partial_{\rho} \sqrt{g}\left(1-\frac{W^{2}(\boldsymbol{\nabla} \Phi)^{2}}{4 f(\Phi)}\right)^{m} \partial_{\rho} \\
& -\frac{1}{\sqrt{g}} \partial_{\rho} \frac{1}{\sqrt{g}} \frac{\Phi_{, s}}{\Phi_{, \rho}} \partial_{s}-\frac{1}{\sqrt{g}} \partial_{s} \frac{1}{\sqrt{g}} \frac{\Phi_{, s}}{\Phi_{, \rho}} \partial_{\rho} \\
& +\frac{1}{\sqrt{g}} \partial_{\rho} \frac{1}{\sqrt{g}} \frac{\Phi_{, s}^{2}}{\Phi_{, \rho}^{2}} \partial_{\rho}+O(W)
\end{aligned}
$$

Given that $\Phi^{(0)}$ is a function of $\rho$ only, we realize that the 
third and fourth terms on the right-hand side are $O(W)$, containing derivatives with respect to $s$ of $\Phi$, the fifth is even $O\left(W^{2}\right)$, so these terms may be dropped immediately in an expansion up to $O(1)$. The second term on the right-hand side owes its existence to the fact that $Q$ is not exactly the projection operator on $\hat{\mathbf{n}}$ and it has a prefactor of $1 / W^{2}$ due to the double derivative in $\rho$. This term which is desirable at leading order, because without it we would not have a determinate zeroth-order solution $\Phi^{(0)}$, is somewhat disturbing at the next order. Since the order of this term is $O\left(W^{m-2}\right)$, we can make it small by choosing $m \geq 3$, i.e., restricting ourselves to even $m$ for the reasons discussed before, we set $m=4$. Then the only remaining term on the right-hand side of Eq. (62) up to order $W^{0}$ is the first term, which is the desired surface Laplacian.

Using this result, we can write the next-to-leading (nontrivial) order inner equation

$$
\begin{gathered}
-v_{n} \partial_{\rho} \Phi^{(0)}=M \partial_{s s} \delta \tilde{\mu}^{(1)}, \\
\delta \tilde{\mu}^{(1)}=-\mathcal{L} \Phi^{(1)}-\kappa \partial_{\rho} \Phi^{(0)},
\end{gathered}
$$

again with $\mathcal{L}$ as given in Eq. (38).

Note that we actually seem to have skipped orders here. The leading-order inner equation is formally $O\left(W^{-4}\right)$, but once the zeroth-order inner solution is fixed, the differential operator $\boldsymbol{\nabla} \cdot Q^{m} \boldsymbol{\nabla}$ is, according to Eq. (62), of order $W^{\min (0, m-2)}$ only, so the order $W^{-3}$ vanishes identically. The order $W^{-2}$ is satisfied automatically, because the zeroth-order chemical potential is zero; the next nontrivial order is $W^{-1}$. Alternatively, one may say that the effective leading order has become $W^{-2}$.

The total linear operator in front of $\Phi^{(1)}$ becomes $-\partial_{s s} \mathcal{L}$. It is Hermitian, because its Hermitian factors commute. Hence $\partial_{\rho} \Phi^{(0)}$ is a left null eigenfunction. Multiplying Eq. (63) from the left by it, integrating with respect to $\rho$ from $-\infty$ to $\infty$, we obtain Eq. (46). This proves that the considered phase-field model based on a modified tensorial mobility has the correct asymptotic behavior for small $W$, neither overconstraining the system by adding, nor leaving it indeterminate by losing equations.

Clearly, Eq. (57) establishes a local conservation law for $\phi$, i.e., the rate of change of the integral of $\phi$ over some control volume is given by the integral of the current $\mathbf{j}$ associated with $\phi$ over the surface of the volume, and this holds for arbitrarily small volumes. $\phi$ is the density of a conserved quantity. In particular, for a system with boundaries through which there is no flux, the volume integral of $\phi$ will be conserved. Therefore we will denote the model discussed in this section as the locally conservative tensorial (LCT) model.

\section{B. Globally conservative model}

If one is willing to give up the conservative nature of the phase-field equations themselves and requires the conservation law only in the asymptotic limit, an even simpler construction is feasible.

Consider the model

$$
\begin{gathered}
\frac{\partial \phi}{\partial t}=M \boldsymbol{\nabla} \cdot P \boldsymbol{\nabla} \frac{1}{W^{2}} \delta \tilde{\mu}+N\left[(\hat{\mathbf{n}} \cdot \boldsymbol{\nabla})^{2} \phi-\frac{2}{W^{2}} f^{\prime}(\phi)\right], \\
P=1-\hat{\mathbf{n}}: \hat{\mathbf{n}}, \\
\delta \tilde{\mu}=-W^{2} \nabla^{2} \phi+2 f^{\prime}(\phi),
\end{gathered}
$$

with both $M$ and $N$ positive constants. With $N=0$, this reduces to the tensorial model of Sec. IV. With $N>0$, it can be shown along similar lines as in Sec. III C 1 that the leadingorder outer solution for $\delta \tilde{\mu}$ is unique leading to the solutions $\phi=0$ and 1 , depending on the boundary conditions at infinity. Moreover, the leading-order inner solution with boundary conditions $\lim _{\rho \rightarrow \infty} \Phi^{(0)}=0, \lim _{\rho \rightarrow-\infty} \Phi^{(0)}=1$ can be shown to be unique up to translations along $\rho$ and is given by Eq. (30) after requiring $\rho=0$ to correspond to the value $\frac{1}{2}$ of the phase field.

The role of the $N$ term is only to fix the profile of the phase field at leading order, otherwise it is constructed so as to not affect the normal velocity of the interface. Once $\Phi^{(0)}$ is set, the next-to-leading order inner equation reads

$$
\left(M \partial_{s s}-N\right) \mathcal{L} \Phi^{(1)}=v_{n} \partial_{\rho} \Phi^{(0)}-M \partial_{s s} \kappa \partial_{\rho} \Phi^{(0)},
$$

and since $M \partial_{s s}-N$ commutes with $\mathcal{L}$, we obtain the desired sharp-interface limit again. Our numerical investigations indeed show that the results depend only weakly on the choice of the parameter $N$, even for moderate separation of the length scales. We find $N \leq 2.5 \mathrm{M} / \mathrm{W}^{2}$ to already give satisfactory results - there are only small differences to results obtained when $N$ is two orders of magnitude smaller, i.e., for $N=1.25 \times 10^{-2} \mathrm{M} / \mathrm{W}^{2}$

While the model (64) is asymptotically conservative, it is desirable to have exact global conservation of the phase-field because this will render long-time simulations more reliable. As the model stands, one might be obliged to choose the interface width smaller as the simulation time becomes larger, which is certainly something one would wish to avoid. Therefore even though the violation of phase-field conservation is small and the model would already be useful in its present form, let us look for an improvement restoring global conservation. By this we mean that $\phi$ need not be the density of a conserved quantity, hence its time derivative need not be the divergence of a current, but for no-flux boundary conditions, the total volume integral of $\phi$ should remain conserved. This can of course be achieved via the introduction of a Lagrange parameter:

$$
\frac{\partial \phi}{\partial t}=M \boldsymbol{\nabla} \cdot P \boldsymbol{\nabla} \frac{1}{W^{2}} \delta \tilde{\mu}+N\left[(\hat{\mathbf{n}} \cdot \boldsymbol{\nabla})^{2} \phi-\frac{2}{W^{2}} f^{\prime}(\phi)\right]-\Lambda(\mathbf{r}, t) .
$$

Here, we have allowed $\Lambda$ to depend on $\mathbf{r}$ which gives useful additional freedom for improvement of the model as we shall see immediately. If $\Lambda$ were restricted to being a simple number, it would have to have the value 


$$
\Lambda=\frac{1}{V} \int_{V} d V \frac{\partial \phi_{\mathrm{old}}}{\partial t}=\frac{N}{V} \int_{V} d V\left[(\hat{\mathbf{n}} \cdot \boldsymbol{\nabla})^{2} \phi-\frac{2}{W^{2}} f^{\prime}(\phi)\right],
$$

where $\partial \phi_{\text {old }} / \partial t$ is the time derivative of the phase-field according to Eq. (64) and $V$ is the volume (or area, in 2D) of the system. Since the first term of the right-hand side of Eq. (66) is conservative anyway, it drops out of the calculation of $\Lambda$, if no fluxes through the system boundary are present. A drawback of the formulation (67) is that it would lead to a modification of the phase field in the bulk from the equilibrium values 0 and 1 , as soon as the Lagrange multiplier became nonzero. This can be avoided by taking advantage of the liberty to make $\Lambda$ vary in space (i.e., we consider a whole set of Lagrange multipliers, not just one). If we take $\Lambda$ of the form

$$
\Lambda(\mathbf{r}, t)=N \frac{|\boldsymbol{\nabla} \phi|}{\int_{V} d V|\boldsymbol{\nabla} \phi|} \int_{V} d V\left[(\hat{\mathbf{n}} \cdot \boldsymbol{\nabla})^{2} \phi-\frac{2}{W^{2}} f^{\prime}(\phi)\right],
$$

the global conservation law is restored without any modification of the bulk solutions. We will call the model described by Eqs. (66) and (68) the globally conservative tensorial (GCT) model.

Of course, we have to verify that the introduction of the Lagrange parameter does not destroy the asymptotic validity of the model. Clearly, the parameter disappears from the leading order of the equation; but the interface velocity is determined at next-to-leading order, and in general one would expect $\Lambda(\mathbf{r}, t)$ to contribute to the equation at that order. This turns out not to be the case and is due to the judicious choice of the form of the parameter, as will be seen below.

The next-to-leading order inner equation can be written

$$
\begin{aligned}
& \left(M \partial_{s s}-N\right) \mathcal{L} \Phi^{(1)}+N \frac{\partial_{\rho} \Phi^{(0)}}{\int d V \partial_{\rho} \Phi^{(0)}} \int d V \mathcal{L} \Phi^{(1)} \\
& =v_{n} \partial_{\rho} \Phi^{(0)}-M \partial_{s s} \kappa \partial_{\rho} \Phi^{(0)},
\end{aligned}
$$

and we are in the awkward situation that the linear operator acting on $\Phi^{(1)}$ is not self-adjoint, so the application of Fredholm's alternative becomes nontrivial. However, the equation contains several terms $\propto \partial_{\rho} \Phi^{(0)}$, which suggests to have $\mathcal{L}$ act on it, leading first to the much simpler equation

$$
\mathcal{L}\left(N-M \partial_{s s}\right) \mathcal{L} \Phi^{(1)}=0,
$$

because $\mathcal{L} \partial_{\rho} \Phi^{(0)}=0$; but here the operator acting on $\Phi^{(1)}$ is semipositive, the operator sandwiched by the two $\mathcal{L}$ s strictly positive. Hence we may conclude that $\mathcal{L} \Phi^{(1)}=0$; but then the left-hand side of Eq. (69) is zero, meaning that the linear equation is in fact homogeneous and the right-hand side has to vanish, too. This implies

$$
v_{n}=M \partial_{s s} \kappa,
$$

the sought-for asymptotic result for the interface velocity. It also implies that the Lagrange multiplier is $O(1)$, instead of $O\left(W^{-1}\right)$, i.e., it is by a factor of the order of $(W \kappa)^{2}$ smaller than the leading-order terms of the equation. This supports what we can point out on the basis of numerical studies: for reasonable separation of length scales as they appear in typical simulations, the influence of the Lagrange parameter is negligibly small.

\section{NUMERICAL SIMULATIONS}

To determine growth rates numerically, we simulate the temporal evolution of a planar front subject to a sinusoidal perturbation. This allows the empirical determination of the main branch of the dispersion relations of the models considered. Moreover, to assess the behavior of the models in a growth situation, we include the coupling to elastic fields, i.e., we simulate the Grinfeld instability. This is easily achieved by replacing the "free" chemical potential $\delta \tilde{\mu}$ with one that includes the correct elastic energy contribution.

In particular, we set

$$
\delta \widetilde{\mu}\left(\nabla^{2} \phi, \phi\right) \equiv-W^{2} \nabla^{2} \phi+2 f^{\prime}(\phi)+\frac{W}{3 \gamma} h^{\prime}(\phi) V_{\mathrm{el}},
$$

with

$$
V_{\mathrm{el}}=(G-\widetilde{G}) \sum_{i, j} u_{i j}^{2}+\frac{\lambda-\tilde{\lambda}}{2}\left(\sum_{k} u_{k k}\right)^{2},
$$

where $G=E /[2(1+\nu)]$ is the shear modulus or first Lamé constant, $\lambda=E \nu /[(1+\nu)(1-2 \nu)]$ the second Lamé constant in the solid phase, and $\widetilde{G}$ and $\tilde{\lambda}$ are the corresponding quantities in the second phase. If the second phase is a liquid, $\widetilde{G}=0$, if it is vacuum, $\widetilde{G}=\tilde{\lambda}=0$. From now on, we will focus on the vacuum case, as it is particularly interesting for diffusion along a free surface.

By $u_{i}$, we denote the displacement field. In addition, equations for the strains $u_{i j}=\frac{1}{2}\left(\partial u_{i} / \partial x_{j}+\partial u_{j} / \partial x_{i}\right)$ have to be provided, which are given by the mechanical equilibrium conditions for the generalized stress tensor $\widetilde{\sigma}_{i j}$,

$$
\sum_{j} \frac{\partial \widetilde{\sigma}_{i j}}{\partial x_{j}}=0, \quad \widetilde{\sigma}_{i j}=h(\phi) \sigma_{i j} .
$$

The function $h(\phi)=\phi^{2}(3-2 \phi)$ interpolates between the two phases-it may be interpreted as the local volume fraction of the solid. $\sigma_{i j}$ is related to $u_{i j}$ via Hooke's law

$$
\sigma_{i j}=\frac{E}{1+\nu}\left(u_{i j}+\frac{\nu}{1-2 \nu} \sum_{k} u_{k k} \delta_{i j}\right) .
$$

That this model produces the right coupling to elasticity in the sharp-interface limit has been shown in various places, e.g., in [5] and [17]. A similar modification of $\delta \hat{\mu}$ leads to the fully coupled RRV model [11].

To simulate the Grinfeld instability in a strip geometry, a dimensionless driving force $F$ is defined as 


$$
F=\frac{\delta^{2}(\lambda+2 G)}{4 \gamma L},
$$

with $\delta$ being a fixed displacement by which the strip is elongated in the direction parallel to the interface. We use a square system of length $L$ and uniform grid spacing $\Delta x$. The interface width, a purely numerical parameter, is chosen to be $W=5 \Delta x$. For a sinusoidal perturbation of a uniaxially strained surface by $\delta y(x)=A_{0} \sin (k x)$ we use a fixed wave number $k L=4 \pi$ and a small amplitude $A_{0} k=\pi / 20$. To obtain a good separation of the characteristic wavelength of the pattern and the interface width, we use $k W=0.16$. The imposed uniaxial stress is given in the figure caption for each case. After having determined the maximum admissible time step, as discussed below, we take as simulation time step $\Delta t=5$ $\times 10^{-4}(\Delta x)^{4} / M$ for the scalar models and $\Delta t=5$ $\times 10^{-3}(\Delta x)^{4} / M$ for the tensorial models. The Poisson ratio is chosen to be $\nu=\lambda /[2(\lambda+G)]=1 / 3$. For the GCT model, we use $N=1.25 \mathrm{M} / \mathrm{W}^{2}$, and for the SM model the standard choice $\tilde{M}(\phi)=36 M \phi^{2}(1-\phi)^{2}$. To minimize the influence of the boundaries, we use helical boundary conditions in the $x$ direction for the displacement fields, i.e., $u_{x}(L, y)=u_{x}(0, y)$ $+\delta, u_{y}(L, y)=u_{y}(0, y)$.

Introducing the Griffith length $L_{G}$,

$$
L_{G}=\frac{L(1-\nu)^{2}}{8 F(1-2 \nu)},
$$

we can write the dispersion relation, i.e., the spectrum of the linear stability operator, as follows:

$$
\omega=M\left(\frac{k^{3}}{L_{G}}-k^{4}\right),
$$

meaning that we have unstable modes at small $k\left(k<1 / L_{G}\right)$ and stable ones at large $k\left(k>1 / L_{G}\right)$. To obtain the spectrum numerically, we vary $L_{G}$ in the simulations.

We use the same algorithm as in [19], which even works for dynamic elasticity; but since we investigate the beginning of the Grinfeld instability, the observed interface velocities are very small in comparison to the speed of sound, and the equations effectively reduce to the static elastic case of Eq. (74).

While the SM model can be discretized in a relatively straightforward manner, some care has to be taken in the other models to avoid divisions by zero. This is rather harmless in the GCT model, where the problem only arises in the computation of the vectors $\hat{\mathbf{n}}(|\boldsymbol{\nabla} \phi|$ goes to zero far from the interface but is positive otherwise, so it is sufficient to ensure that the denominator of $\hat{\mathbf{n}}$ does not become smaller than a small positive number). The main requirement in the RRV model is that $g(\phi)$ should not be set exactly equal to zero.

In the LCT model, more attention has to be paid to the situation where $f(\phi)$ becomes small, as will be discussed below. Essentially, we make four types of comparison. First, we compare the time evolution of sinusoidal fronts (initialized with the correct width of the profile) for a number of imposed uniaxial stresses and obtain the linear stability spectrum numerically. Second, we increase the time step in the simulation for given mesh size until we reach the maximum

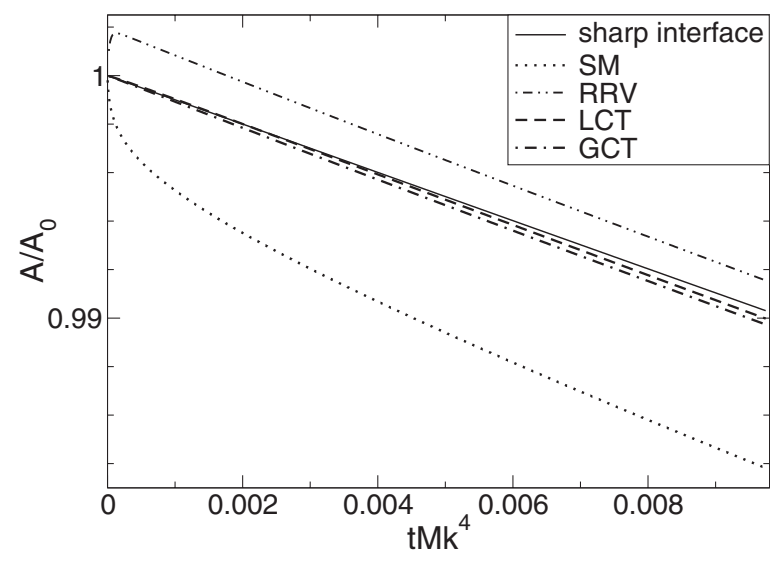

FIG. 1. Amplitude evolution for a uniaxial stress of $F=0$, i.e., a Griffith length $L_{G}=\infty$.

possible time step providing convergence to the correct interface dynamics and then compare the achieved values. Next, we initialize a planar profile with the wrong interface width and observe relaxation to a profile of the correct width. In a realistic simulation, slight deviations from the correct profile width may easily appear in an initial condition for a curved interface, as analytical expressions for constant-width profiles at arbitrary curvature are not readily available (even for an initial germ with a shape as simple as an ellipse it is not quite trivial to give such an expression). Any phase-field code should be robust against these local variations of the profile width and should have it relax to the correct value. Finally, we look at the evolution of an elliptical inclusion. Since the phase-field parameter is a conserved quantity, the ellipse should morph to a circle with the same area.

In Figs. 1-3, we show the temporal evolution of a sine profile starting with a prescribed amplitude for different values of the imposed uniaxial stress. The four models are compared directly with the sharp interface prediction resulting from Eq. (78). Figure 1 exemplifies the stress-free case discussed analytically.

All the situations considered correspond to either weak decay or weak growth of the amplitude, as the expected exponential behavior still appears linear on the considered time scale. We note that all the models agree with the predicted behavior of the sharp-interface limit to within better than $1 \%$

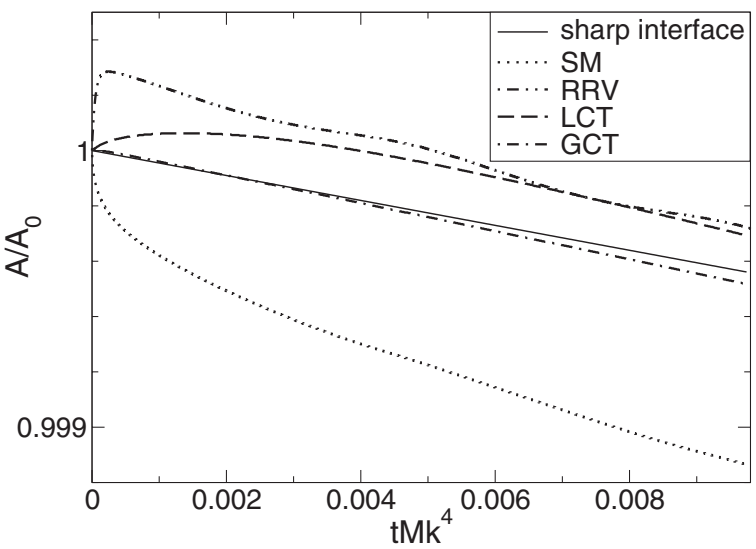

FIG. 2. Amplitude evolution for a uniaxial stress of $F=2$. 


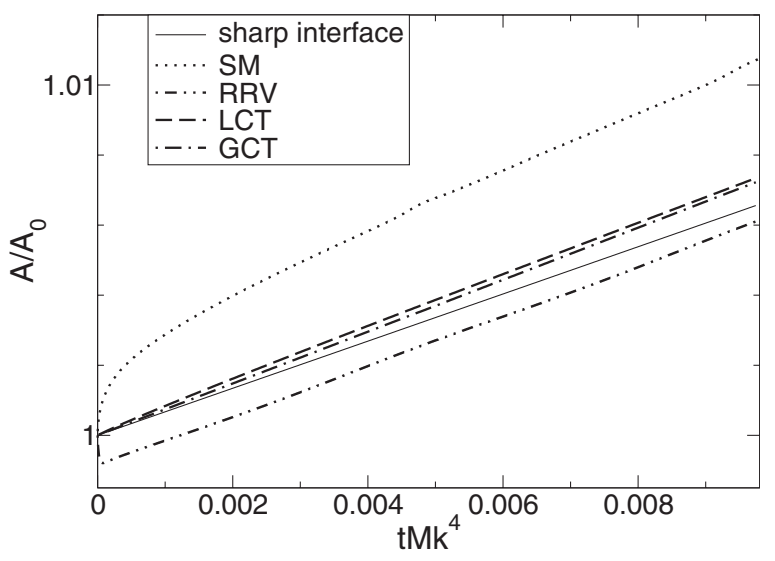

FIG. 3. Amplitude evolution for a uniaxial stress of $F=3.5$.

for our parameters and time span. While it may be observed unambiguously that the SM model displays the largest deviation from the desired result, one may find it surprising that it reproduces the limit so well after all, taking into account that it does not have the right asymptotics. Presumably those equations of the asymptotic behavior to which the system can adjust locally act as an attractor for the dynamics even before the full set of equations, implying more global restrictions such as Eq. (47), becomes active. It is striking that this seems to work even in a growth situation, where interface velocities increase on average.

Figure 4 gives a comparison of the linear stability spectra, obtained by simulation of the four models, with the analytical expression Eq. (78) of the sharp-interface model. It is pretty clear that the SM model is farthest off the correct value both below and above the fastest-growing wave number. The LCT model is good for wave numbers above that of the fastest-growing mode but shows stronger deviations than both the RRV and GCT model below that mode. The latter two models are about equally close to the correct spectrum throughout the whole wave-number domain.

Figure 5 displays the maximum time step for a given grid spacing leading to smooth growth where the results for all models agreed perfectly, independent of the timediscretization. We observe, for all models, a scaling of the maximum admissible time step as $\Delta t \sim \Delta x^{4} / M$, which is not too surprising given the fact that the equations simulated are

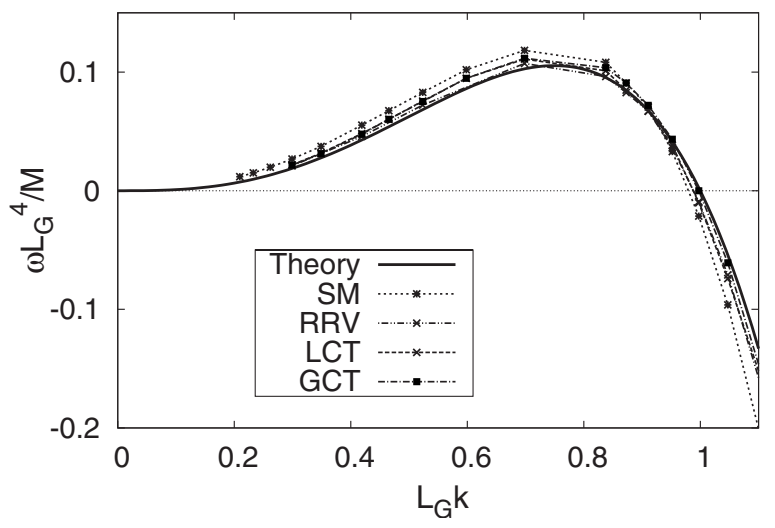

FIG. 4. Full spectrum of the ATG instability.

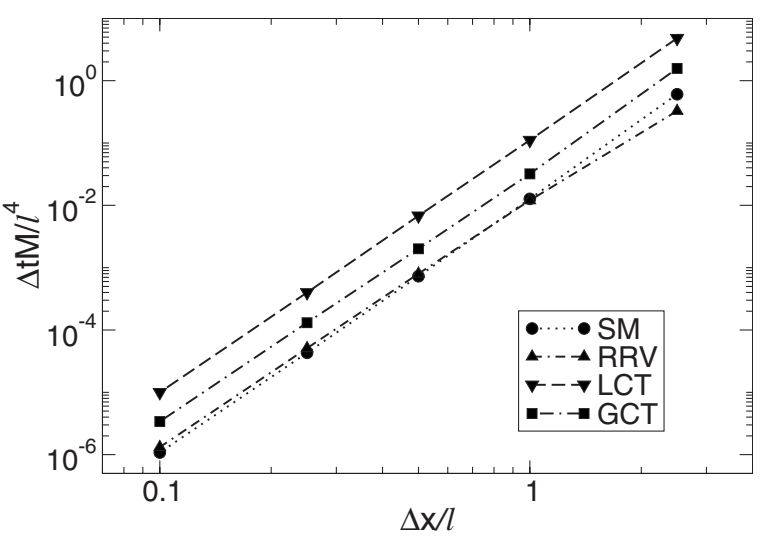

FIG. 5. Allowable maximal time step $\Delta t$ as a function of mesh size, where $l$ is an arbitrary length unit. Lines are a guide for the eyes only.

fourth order in space and first order in time, and we used straightforward explicit schemes for discretization. However, while in the two scalar models (SM, RRV) about the same maximum time step is possible, the tensorial models allow larger time steps; a simulation with the LCT model gains a factor of about 10 in time steps over the scalar models. While by use of adaptive mesh techniques [11] and implicit schemes, the overall running time can certainly be reduced by more than this factor for large systems, the advantage of the tensorial models may persist even in such a setting as it is consistently present in a range of grid spacings. We suspect that the advantage in stability of the explicit scheme will turn into an advantage of accuracy when the discretization is made implicit. Clearly, a verification (or falsification) of this idea will require simulations of the RRV, LCT, and GCT models using implicit discretization schemes, which may raise additional technical issues for the nonlocal GCT model.

Next, it is interesting to compare how the different models behave regarding their relaxation to a stationary profile when initialized with a straight interface having a width that is either too small or too large. These simulations are done without elasticity, i.e., for $F=0$. First, we verify that all the models remain in their equilibrium state when initialized with a tanh profile of the correct width.

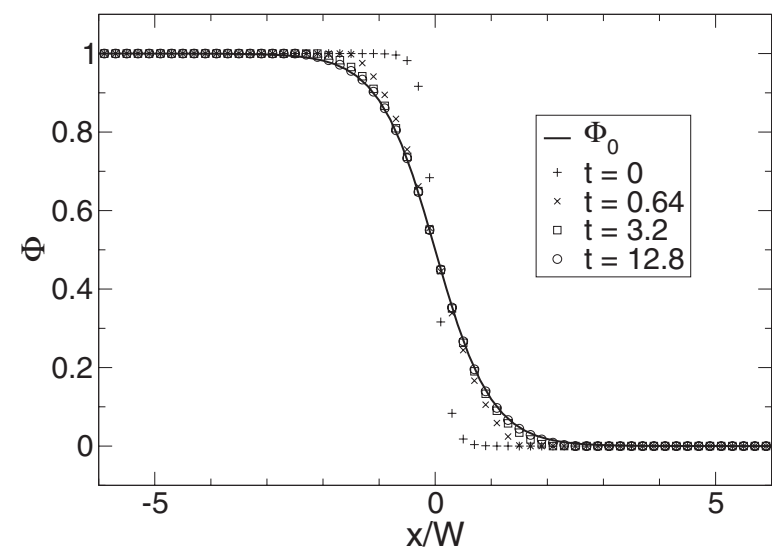

FIG. 6. Relaxation toward the correct interface profile for the $\mathrm{SM}$ model. The initial interface width is $0.25 \mathrm{~W}$, the time $t$ is given in units of $W^{4} / M$. 


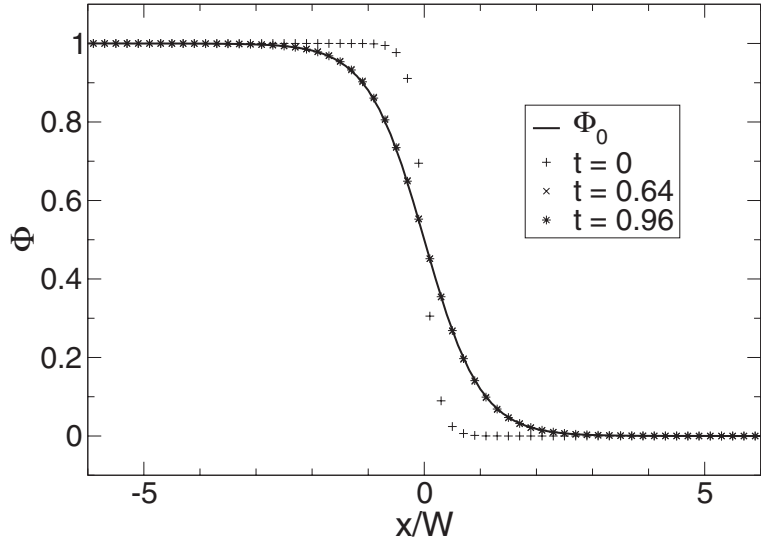

FIG. 7. Relaxation toward the correct interface profile for the RRV model. The initial interface width is $0.25 \mathrm{~W}$, the time $t$ is given in units of $W^{4} / M$.

For brevity of language, we define here a profile $\tanh z / W$ to have width $W$, even when the width on which it rises from -0.9 to 0.9 rather is $2.94 \mathrm{~W}$. In all plots, the time $t$ is given in units of $W^{4} / M$, and only a small section about the interface is shown.

All of the models do reasonably well in relaxing from a planar profile of width $0.25 \mathrm{~W}$ to their equilibrium state, see Figs. 6-9. In our implementation and with the given sets of parameters, simulations with the RRV model broke down if the initial interface width was chosen to be smaller than $0.23 \mathrm{~W}$. The RRV and GCT model relax quickly to a final profile of width $W$, while the SM model needs a little more time (but the permissible time step is larger for the GCT model, so the numerical running time is shortest for it) and the LCT model is slowest in terms of simulated time (but not in terms of time steps).

Some care has to be taken in the discretization to make the LCT model deal efficiently with too thin interfaces, as shall be discussed now. We write $Q^{4}=1-\hat{\mathbf{n}}: \hat{\mathbf{n}}+b_{0}^{4} \hat{\mathbf{n}}: \hat{\mathbf{n}}$ with

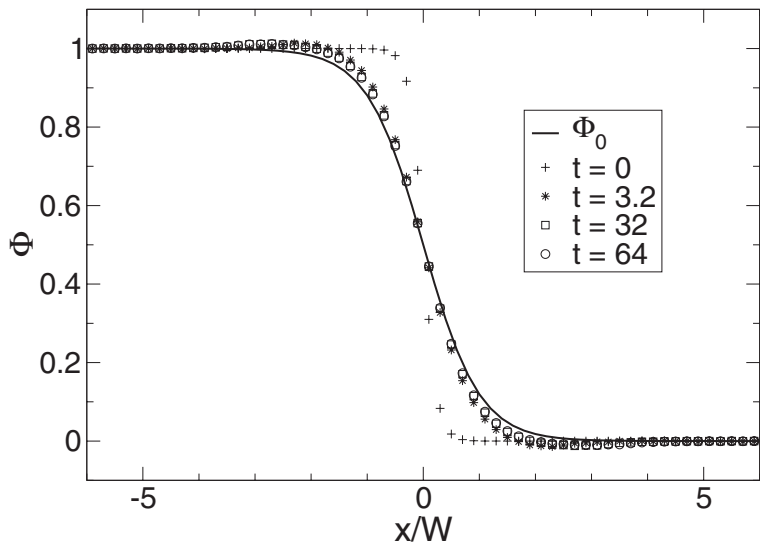

FIG. 8. Relaxation toward the correct interface profile for the LCT model. The initial interface width is $0.25 \mathrm{~W}$, the time $t$ is given in units of $W^{4} / M$.

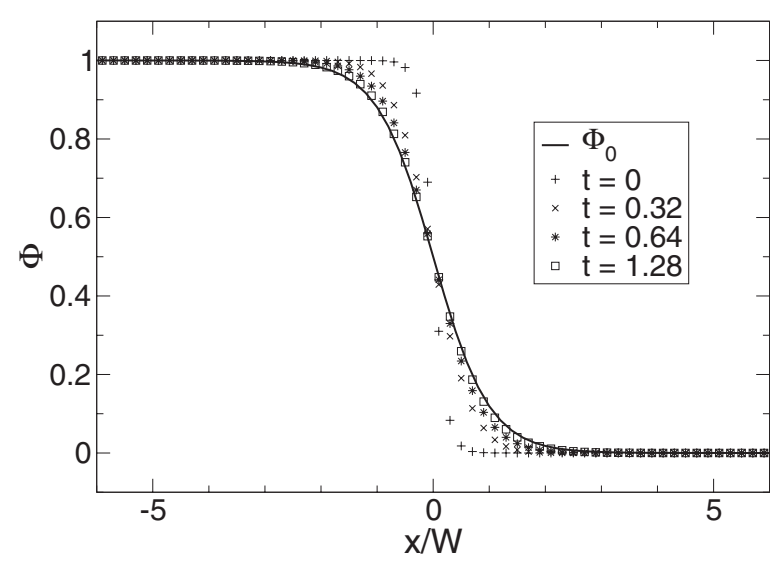

FIG. 9. Relaxation toward the correct interface profile for the GCT model. The initial interface width is $0.25 \mathrm{~W}, N=1.25 \mathrm{M} / \mathrm{W}^{2}$, the time $t$ is given in units of $W^{4} / M$.

$$
b_{0}=1-\frac{W^{2}(\nabla \phi)^{2}}{4 f(\phi)} .
$$

Taking an interface of width $\xi$ with the profile $\phi(z)=\frac{1}{2}(1$ $-\tanh z / \xi)$ we find

$$
b_{0}^{4}=\left(1-\frac{W^{2}}{\xi^{2}}\right)^{4},
$$

which becomes very large for $\xi \ll W$. In fact, for $\xi=0.25 \mathrm{~W}$, we have $b_{0}^{4}=50625$, a number that, when plugged into the equations of motion, would impose a prohibitively small time step for stability (or accuracy, in an implicit scheme). Hence we introduce a cutoff for $b_{0}^{4}$ on the order of 50 . In production runs, where one normally starts with a front profile having at least approximately the correct width, a cutoff of 10 may be sufficient, which will accelerate relaxation of too thin an interface quite a bit.

When the profile is initialized with too large a width [Figs. 10-13], more interesting differences can be seen. Not unexpectedly, the GCT model [Fig. 13] is the one making the least fuss about an interface five times too wide: that the model is nonconservative on the scale where the phase-field

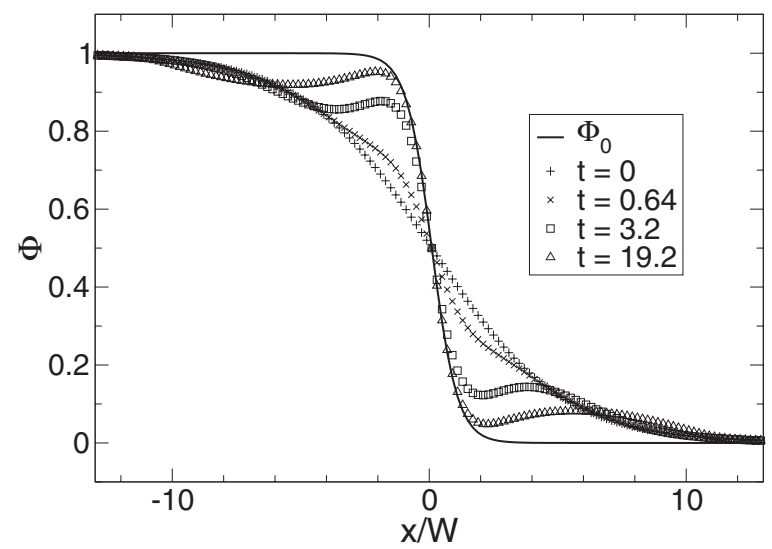

FIG. 10. Relaxation toward the correct interface profile for the SM model. The initial interface width is $5 W$, the time $t$ is given in units of $W^{4} / M$. 


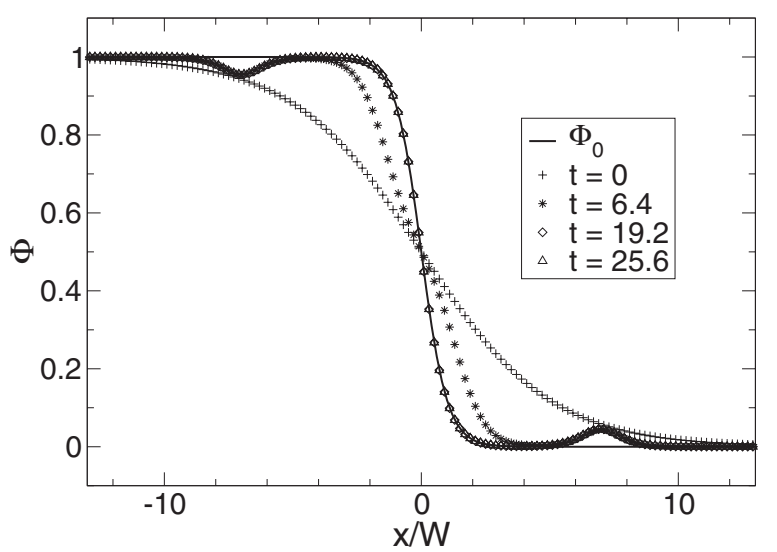

FIG. 11. Relaxation toward the correct interface profile for the RRV model. The initial interface width is $5 W$, the time $t$ is given in units of $W^{4} / M$.

varies strongly is an advantage here. The interface approaches its correct width in a time of about $t \approx 1.25 W^{2} / N$, which corresponds to $t \approx 1 W^{4} / M$ for our parameter choice. For the other models, this takes much longer, as this kind of adaptation requires diffusion orthogonally to the front, which is slow because it is suppressed in the asymptotic limit.

The RRV model goes through a series of transformations of the profile involving as an intermediate state a spatially varying slope in the vicinity of the contour line $\phi=\frac{1}{2}$ defining the interface position. Even after a time of $t \approx 30 W^{4} / M$, while near the interface position the profile is well-behaved and has the right width, there are still indentations in it far from the interface, and these disappear only slowly.

While the LCT model keeps a nicer profile all the time, it relaxes only slowly as well. Moreover, if the boundary values of $\phi$ are not fixed to be equal to zero or one, it will relax to constant values in the bulk different from these ideal values (in the absence of elasticity). Indeed, inspection of Eq. (57) shows immediately that any constant value of $\phi$ solves the bulk equations of motion. (This is no longer true in the presence of elasticity.) For phases extending to the system boundary, the value of the constant is only fixed by the boundary conditions. Therefore the model should always be

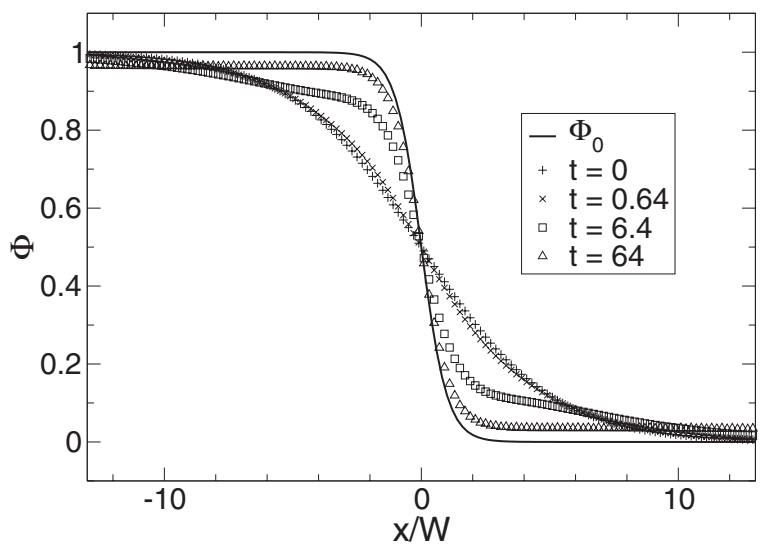

FIG. 12. Relaxation toward the correct interface profile for the LCT model. The initial interface width is $5 \mathrm{~W}$, the time $t$ is given in units of $W^{4} / M$.

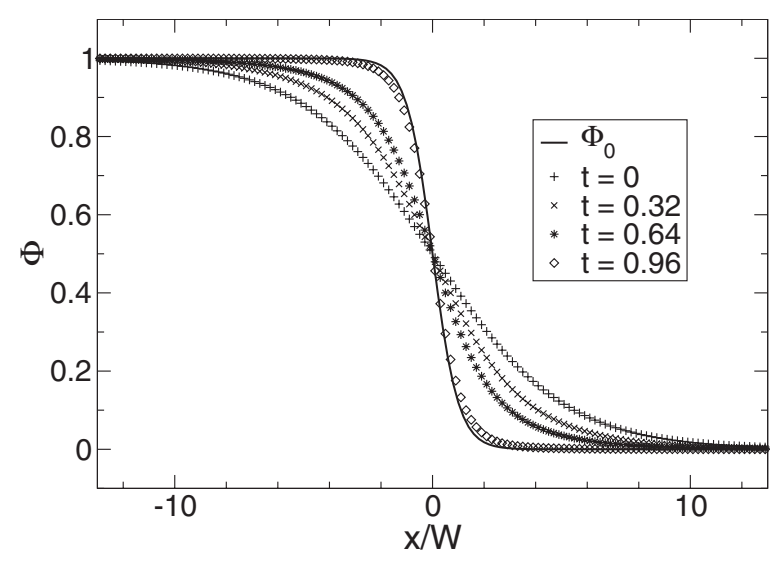

FIG. 13. Relaxation toward the correct interface profile for the GCT model. The initial interface width is $5 W$, the time $t$ is given in units of $W^{4} / M$.

run with Dirichlet boundary conditions for the phase field. (Due to the conservation law, inclusions of one phase in another will keep their $\phi$ value, even in the presence of elasticity, if correctly initialized to zero or one, as long as their inner volume is much larger than that of their interface.) Performing such a simulation, we found relaxation to be as slow as for the SM and RRV models but the interface profile to look more reasonable.

To summarize, when interface thickness is believed to be an issue in simulations, i.e., when there are reasons to think that it might vary considerably (which may be the case when surface tension anisotropy is included in the model), the nonexact realization of the conservation law by the GCT model may turn out a virtue rather than a drawback, since changes in the direction normal to the interface by diffusion only, as realized in the other models, tend to be too slow.

Finally, we compare the different dynamics for a "reallife" situation of an elliptical inclusion that morphs into a circle without elastic effects, $F=0$. The system is initialized with a sharp interface ellipse with semimajor $a_{0}$ and semiminor $a_{0} / 2$ and is then allowed to relax for a few time steps running the GCT model (with a Lagrange multiplier $\Lambda=0$ ), in order to obtain an initial condition with the correct interface width everywhere. We then measure the time evolution of the semimajor and semiminor of the ellipse, continuing the run with the model to be studied.

As Fig. 14 shows, all models but the SM model converge to a circle with the correct radius $\sqrt{2} a_{0} / 2$. The SM model shows different behavior, namely a too small radius that seems to decrease further. Since the phase field is a conserved quantity (we also checked that numerically for our code), this can only mean that the final shape of the inclusion is not a true circle but a slightly deformed one, displaying a certain level of anisotropy. We then increase the size of the system and the included ellipse while keeping the interface width constant, resulting in a better scale separation $a_{0} / W$. While for the LCT, GCT, and RRV models the curves collapse onto a single line, this is not the case for the SM model. Figure 15 demonstrates this behavior for the LCT model. The comparison for the SM model is shown in Fig. 16. 


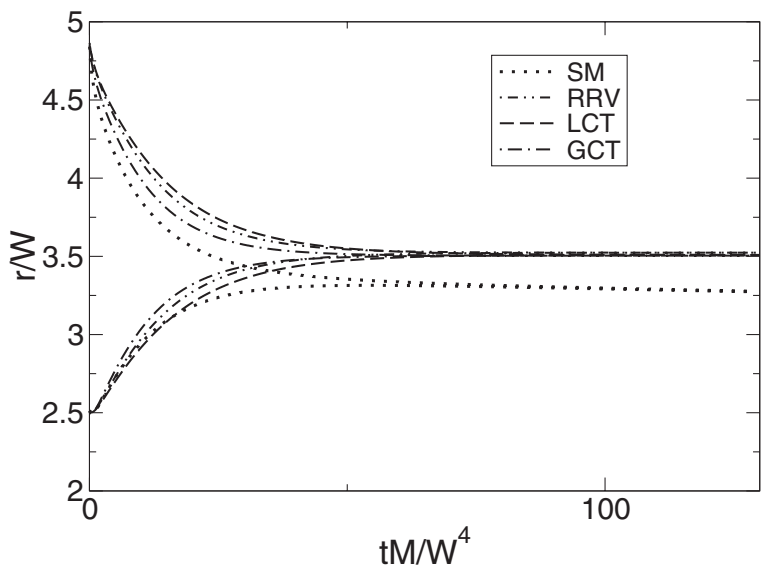

FIG. 14. Comparison of the time development of the size of an elliptical inclusion. The square system had the length $L / W=20$. The initial ellipse had a semimajor of $a_{0}=L / 4$ and a semiminor of $L / 8$. All models except the SM model converge to circles with the same radius $r$.

\section{CONCLUSIONS}

The intuitive approach to constructing a phase-field model for surface diffusion consists of using the chemical potential known from the nonconservative model to define a current, involving its gradient and a mobility that vanishes in the bulk phases, and of taking the divergence of this current as the time derivative of the phase-field. As has been shown in this paper, this approach-the SM model-fails to produce the correct asymptotics in a subtle way. It does reproduce the equilibrium limit correctly and it appears to work numerically, although less efficiently than the alternatives discussed.

We offer a simple argument why the SM model should not be expected to work properly: The chemical potential functional of the model is constructed so that the chemical potential vanishes in the bulk phases. As the diffusion operator is essentially a scalar, diffusion acts also orthogonally to the interface; in its vicinity, the effect is even strong because

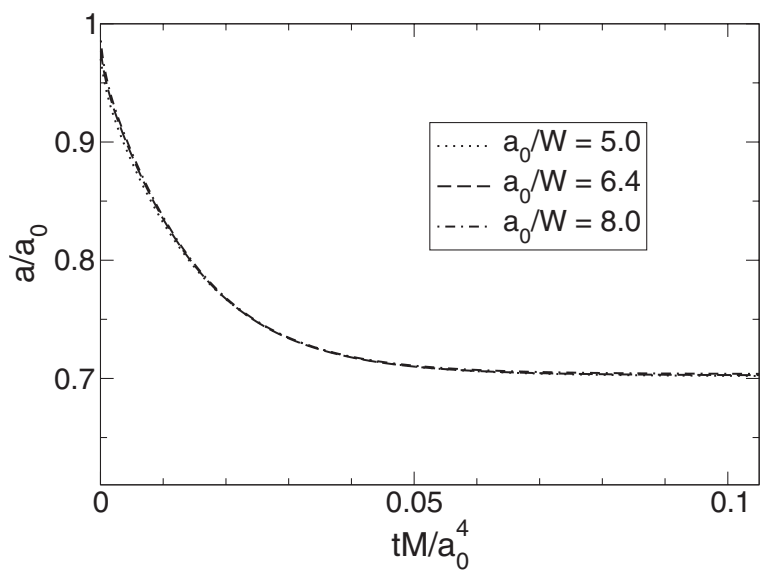

FIG. 15. Elliptical inclusion: comparison of the time development of the length of the semimajor $a$ for the LCT model. The initial length is denoted by $a_{0}$ and the different curves correspond to different scale separations $a_{0} / W$. All curves collapse onto a single line.

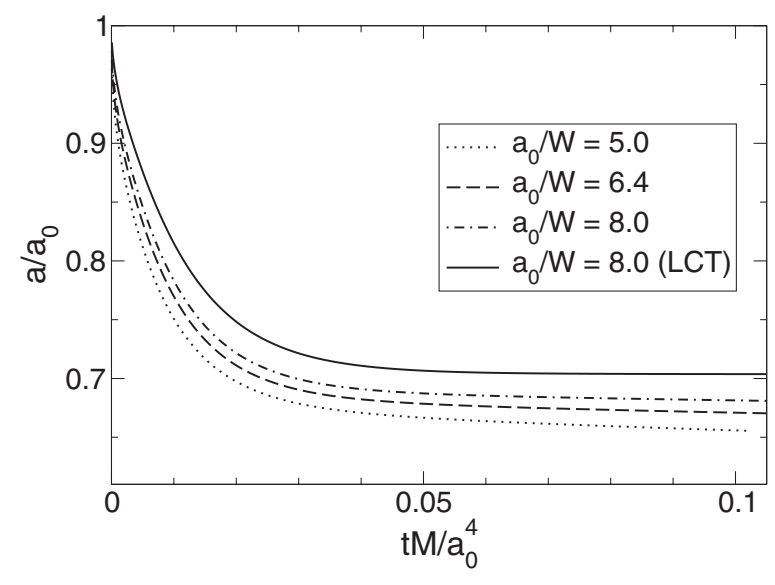

FIG. 16. Elliptical inclusion: comparison of the time development of the length of the semimajor $a$. The initial length is denoted by $a_{0}$ and the different curves correspond to different scale separations $a_{0} / W$. The performance of the SM model becomes asymptotically better for larger systems. Results for the LCT model have been included as a reference.

the slope of the phase-field is largest in the direction perpendicular to the corresponding level set. This diffusive effect constitutes a driving force for relaxation of the chemical potential toward zero also close to the interface (asymptotically, the chemical potential is zero at next-to-leading order). Surface diffusion of the chemical potential is then not the only effect contributing to the interface dynamics.

The RRV model avoids this problem by leaving the chemical potential in the bulk undetermined. Absence of diffusion in the bulk is not guaranteed by the chemical potential but by the vanishing mobility. Since the bulk chemical potential is free to vary, a true interface chemical potential can build up, the surface diffusion of which governs the interface dynamics.

Our contribution in this paper is to explore the idea that the failure of the SM model might be remedied instead by making the mobility a tensor. After all, surface diffusion may be interpreted as highly anisotropic three-dimensional diffusion with a diffusion tensor that has a zero eigenvalue in one direction. Whereas the preexisting RRV model has the correct asymptotics, it does not exploit that idea. A straightforward attempt of its realization, however, fails in a rather drastic way, because restricting diffusion to the surfaces of constant phase-field does not impose any functional dependence of $\phi$ in the normal direction given by this foliation.

Modifying the tensorial mobility, one obtains the LCT and GCT models, both exhibiting the correct asymptotic behavior. Numerical study of the four models suggests that whereas the SM model has a range of quantitative validity (despite its not being asymptotic), the other models indeed faithfully capture the correct asymptotic behavior. The RRV and LCT model are strictly conservative, the GCT model has the advantage of higher robustness.

The tensorial models remain stable for larger time steps than the scalar ones in explicit discretization schemes. Whether this will lead to an advantage in accuracy affording larger time steps also in implicit schemes is a tempting speculation (supported by consideration of the orders at 
which the asymptotic results appear), but at the moment this is simply an open question, decidable only by future simulations. However, we believe that our presentation shows the models to hold some promise and invites one to put them to further tests.

\section{ACKNOWLEDGMENTS}

We wish to thank E. Brener and H. Müller-Krumbhaar for stimulating discussions that have led, among other things, to the introduction of a Lagrange parameter into the GCT model. Support of this work by DFG Grants No. KA 672/9-1 and No. MU 1170/6-1 and the U. S. Department of Energy, Office of Basic Energy Sciences, through the Computational Materials Science Network program is gratefully acknowledged.

\section{APPENDIX A: MATCHING CONDITIONS}

Let $\widetilde{\psi}(x, z, t)=\psi(r, s, t)$ be some arbitrary (sufficiently often differentiable) function of space and time obtained in solving the outer equations. We write the corresponding function of the inner solution as $\Psi(\rho, s, t)$, and suppress from now on, in this section, the dependence of functions on $s$ and $t$. Moreover, we write the coefficient functions in expansions with respect to $W$ with simple subscripts indicating their order rather than superscripts in parentheses as in the main text. There, the notation is dictated by the fact that a subscript would interfere with certain other subscripts; here, a superscript would interfere with the primes denoting derivatives.

We must have the asymptotic relationship

$$
\Psi(\rho) \sim \psi(r)=\psi(W \rho) \quad(\rho \rightarrow \infty, W \rightarrow 0, W \rho \rightarrow 0) .
$$

Expanding both functions in powers of $W$, we get

$$
\begin{gathered}
\Psi(\rho)=\Psi_{0}(\rho)+W \Psi_{1}(\rho)+W^{2} \Psi_{2}(\rho)+\cdots, \\
\psi(W \rho)=\psi_{0}(r)+W \psi_{1}(r)+W^{2} \psi_{2}(r)+\cdots \\
=\psi_{0}(0)+W\left[\rho \psi_{0}^{\prime}(0)+\psi_{1}(0)\right] \\
+W^{2}\left[\rho^{2} \frac{1}{2} \psi_{0}^{\prime \prime}(0)+\rho \psi_{1}^{\prime}(0)+\psi_{2}(0)\right]+\cdots,
\end{gathered}
$$

where the derivatives are to be taken for $r \rightarrow+0$, should they be discontinuous at $r=0$. Analogous expressions with $r \rightarrow$ -0 are obtained for the asymptotics as $\rho \rightarrow-\infty$.

Equating powers of $W$, we then successively get the asymptotic relationships

$$
\begin{gathered}
\lim _{\rho \rightarrow \pm \infty} \Psi_{0}(\rho)=\psi_{0}( \pm 0), \\
\Psi_{1}(\rho) \sim \rho \psi_{0}^{\prime}( \pm 0)+\psi_{1}( \pm 0) \quad(\rho \rightarrow \pm \infty),
\end{gathered}
$$

$$
\Psi_{2}(\rho) \sim \frac{1}{2} \rho^{2} \psi_{0}^{\prime \prime}( \pm 0)+\rho \psi_{1}^{\prime}( \pm 0)+\psi_{2}( \pm 0) \quad(\rho \rightarrow \pm \infty) .
$$

Moreover, asymptotic relations such as Eq. (A5) can be decomposed into statements about function limits

$$
\begin{gathered}
\lim _{\rho \rightarrow \pm \infty} \partial_{\rho} \Psi_{1}(\rho)=\psi_{0}^{\prime}( \pm 0), \\
\lim _{\rho \rightarrow \pm \infty}\left[\Psi_{1}(\rho)-\rho \psi_{0}^{\prime}( \pm 0)\right]=\psi_{1}( \pm 0) .
\end{gathered}
$$

\section{APPENDIX B: LEADING ORDER OUTER EQUATION}

We discuss here in more detail the solutions of the leading order outer equation in the SM model; the uniqueness of this solution is important and not entirely trivial, and one might suspect that additional solutions exist, which could render the phase-field model useless. To rule out this possibility, we demonstrate here that the solution of Eq. (25) together with the proper boundary conditions is indeed uniquely $\phi^{(0)}=1$ or $\phi^{(0)}=0$.

In particular, since we expect the outer solution to have jump discontinuities, we will assume that $\phi^{(0)}$ is only piecewise continuously differentiable. If we take the positions of its jump discontinuities [i.e., of the interface(s) or transition region(s)] between phases as known, it is not difficult to construct a proof.

Equation (25) is a continuity equation for the current $\mathbf{J}_{0}$ $\equiv-\tilde{M} \boldsymbol{\nabla} f^{\prime}\left(\phi^{(0)}\right)$. By inspection of Eq. (21) or, more explicitly, the leading-order inner equation (26), we note that there is no surface current at leading order (the current does not have a tangential component, which would be connected with a derivative with respect to $s$ ), so we may use a Gaussian pillbox construction to demonstrate that $\mathbf{J}_{0}$ is continuous across any two-phase interface and hence in all of space.

Next, we multiply Eq. (25) by $f^{\prime}\left(\phi^{(0)}\right)$ and integrate over all of space. Then we employ Gauss's theorem in each of the subvolumes, into which space is compartmentalized by the interface(s).

(We will often use the nomenclature for three-dimensional systems, not distinguishing between surface integrals and boundary contour integrals; also we employ $d V$ for the volume element of both two- and three-dimensional space.)

Concisely written, the theorem states

$$
\begin{aligned}
0 & =\int d V f^{\prime}\left(\phi^{(0)}\right) \boldsymbol{\nabla} \cdot \tilde{M} \boldsymbol{\nabla} f^{\prime}\left(\phi^{(0)}\right) \\
& =\int_{\partial V} d S \mathbf{N} \cdot f^{\prime}\left(\phi^{(0)}\right) \tilde{M} \nabla f^{\prime}\left(\phi^{(0)}\right)-\int d V \tilde{M}\left[\nabla f^{\prime}\left(\phi^{(0)}\right)\right]^{2},
\end{aligned}
$$

where $d S$ is the surface element and $\mathbf{N}$ stands for the outer normal on the surface $\partial V$ of the (infinite) volume considered. In general, this volume will consist of several disjoint pieces. So $\partial V$ contains outer surfaces (at infinity) and inner ones (at the phase boundaries). 
With our boundary condition at infinity, the contribution by the outer surfaces vanishes (this also holds for von Neumann boundary conditions $\mathbf{N} \cdot \boldsymbol{\nabla} \phi^{(0)}=0$. Each of the inner surfaces appears twice, once on each side of the phase transition region(s).

The current appearing in the surface integrals of Eq. (B1) is not $\mathbf{J}_{0}$ but $f^{\prime}\left(\phi^{(0)}\right) \mathbf{J}_{0}$. It is, however, easy to convince oneself that continuity of $\mathbf{J}_{0}$ implies continuity of $f^{\prime}\left(\phi^{(0)}\right) \mathbf{J}_{0}$. For $f^{\prime}\left(\phi^{(0)}\right)$ can have jump discontinuities at most. Wherever $f^{\prime}\left(\phi^{(0)}\right)$ is discontinuous, the factor $\boldsymbol{\nabla} f^{\prime}\left(\phi^{(0)}\right)$ of $\mathbf{J}_{0}$ has a surface $\delta$ function singularity. Since $\mathbf{J}_{0}$ itself is continuous, this means that $\tilde{M}$ must be zero at these positions, if any; but then the factor $\tilde{M}$ will regularize the singularity of the combination $f^{\prime}\left(\phi^{(0)}\right) \nabla f^{\prime}\left(\phi^{(0)}\right)$ as well. [In reality, $f^{\prime}\left(\phi^{(0)}\right)$ remains continuously differentiable (see below), so $\tilde{M}$ is not restricted.]

Since $f^{\prime}\left(\phi^{(0)}\right) \tilde{M} \nabla f^{\prime}\left(\phi^{(0)}\right)$ is continuous across the interface(s), the contributions of the pairwise inner surfaces cancel each other because their normal vectors are oppositely oriented. [If we consider transition regions instead of interfaces, we may conclude the total sum of the surface integrals to be $O(W)$, so we can drop them from the zeroth-order consideration.] The outcome of these deliberations is that we can state

$$
\int d V \tilde{M}\left[\nabla f^{\prime}\left(\phi^{(0)}\right)\right]^{2}=0 .
$$

If $\tilde{M}$ is positive almost everywhere, we immediately get $f^{\prime}\left(\phi^{(0)}\right)=$ const, and the boundary conditions require the constant to be zero. (A constant is of course a continuously differentiable function, see our remark above.) This conclusion remains unchanged, if $\tilde{M}$ becomes zero only when $\phi^{(0)}$ is zero or one-a standard choice [16] is $\tilde{M}(\phi) \propto \phi^{2}(1-\phi)^{2}$.

Hence the unique solution to the leading-order outer problem is, if we now consider it an equation for $\phi^{(0)}$ again, $\phi^{(0)}=1$ in $\Omega^{-}$and $\phi^{(0)}=0$ in $\Omega^{+}$, where $\Omega^{\mp}$ are those regions of space, separated by the interface(s), in which $\lim _{|\mathbf{r}| \rightarrow \infty} \phi^{(0)}=1$ and 0 , respectively. The solution $\phi^{(0)}=\frac{1}{2}$, still possible for the equation interpreted as an equation for $f^{\prime}\left(\phi^{(0)}\right)$, is excluded by the boundary conditions for $\phi^{(0)}$. (This argument presupposes that we have no domains that are not connected with infinity. For the interior of a closed interface, the solution $\phi^{(0)}=1 / 2$ would have to be excluded by a stability argument or by making reference to initial conditions.)
[1] A. Karma and W.-J. Rappel, Phys. Rev. E 53, R3017 (1996).

[2] A. Karma and W.-J. Rappel, Phys. Rev. E 57, 4323 (1998).

[3] J. Müller and M. Grant, Phys. Rev. Lett. 82, 1736 (1999).

[4] K. Kassner and C. Misbah, Europhys. Lett. 46, 217 (1999).

[5] K. Kassner, C. Misbah, J. Müller, J. Kappey, and P. Kohlert, Phys. Rev. E 63, 036117 (2001).

[6] M. Grinfeld, Sov. Phys. Dokl. 31, 831 (1986).

[7] R. Asaro and W. Tiller, Metall. Trans. 3, 1789 (1972).

[8] W. H. Yang and D. J. Srolovitz, Phys. Rev. Lett. 71, 1593 (1993).

[9] K. Kassner and C. Misbah, Europhys. Lett. 28, 245 (1994).

[10] M. Haataja, J. Müller, A. D. Rutenberg, and M. Grant, Phys. Rev. B 65, 165414 (2002).

[11] A. Rätz, A. Ribalta, and A. Voigt, J. Comput. Phys. 214, 187 (2006).

[12] D.-H. Yeon, P.-R. Cha, and M. Grant, Acta Mater. 54, 1623 (2006).
[13] G. Caginalp, Phys. Rev. B 38, 789 (1988)

[14] J. W. Cahn, C. M. Elliott, and A. Novick-Cohen, Eur. J. Appl. Math. 7, 287 (1996).

[15] M. Mahadevan and R. M. Bradley, Physica D 126, 201 (1999).

[16] D. N. Bhate, A. Kumar, and A. F. Bower, J. Appl. Phys. 87, 1712 (2000).

[17] K. Kassner, e-print arXiv:cond-mat/0607823.

[18] M. A. Spivak, Comprehensive Introduction to Differential Geometry, 2nd ed. (Publish or Perish, Wilmington, DE, 1979), Vol. 3.

[19] R. Spatschek, C. Müller-Gugenberger, E. Brener, and B. Nestler, Phys. Rev. E 75, 066111 (2007).

[20] M. Bertalmío, L.-T. Cheng, S. Osher, and G. Sapiro, J. Comput. Phys. 174, 759 (2001).

[21] M. Burger, F. Haußer, C. Stöcker, and A. Voigt, J. Comput. Phys. 225, 183 (2007). 